Louisiana State University

LSU Digital Commons

\title{
Continuum Reverberation Mapping of the Accretion Disks in Two Seyfert 1 Galaxies
}

\author{
M. M. Fausnaugh \\ The Ohio State University \\ D. A. Starkey \\ University of St Andrews \\ Keith Horne \\ University of St Andrews \\ C. S. Kochanek \\ The Ohio State University \\ B. M. Peterson \\ The Ohio State University
}

See next page for additional authors

Follow this and additional works at: https://digitalcommons.Isu.edu/physics_astronomy_pubs

\section{Recommended Citation}

Fausnaugh, M., Starkey, D., Horne, K., Kochanek, C., Peterson, B., Bentz, M., Denney, K., Grier, C., Grupe, D., Pogge, R., De Rosa, G., Adams, S., Barth, A., Beatty, T., Bhattacharjee, A., Borman, G., Boroson, T., Bottorff, M., Brown, J., Brown, J., Brotherton, M., Coker, C., Crawford, S., Croxall, K., Eftekharzadeh, S., Eracleous, M., Joner, M., Henderson, C., Holoien, T., Hutchison, T., Kaspi, S., Kim, S., \& King, A. (2018). Continuum Reverberation Mapping of the Accretion Disks in Two Seyfert 1 Galaxies. Astrophysical Journal, 854 (2) https://doi.org/10.3847/1538-4357/aaaa2b

This Article is brought to you for free and open access by the Department of Physics \& Astronomy at LSU Digital Commons. It has been accepted for inclusion in Faculty Publications by an authorized administrator of LSU Digital Commons. For more information, please contact ir@lsu.edu. 


\section{Authors}

M. M. Fausnaugh, D. A. Starkey, Keith Horne, C. S. Kochanek, B. M. Peterson, M. C. Bentz, K. D. Denney, C. J. Grier, D. Grupe, R. W. Pogge, G. De Rosa, S. M. Adams, A. J. Barth, Thomas G. Beatty, A. Bhattacharjee, G. A. Borman, T. A. Boroson, M. C. Bottorff, Jacob E. Brown, Jonathan S. Brown, M. S. Brotherton, C. T.

Coker, S. M. Crawford, K. V. Croxall, Sarah Eftekharzadeh, Michael Eracleous, M. D. Joner, C. B. Henderson, T. W.S. Holoien, T. Hutchison, Shai Kaspi, S. Kim, and Anthea L. King 


\section{Continuum Reverberation Mapping of the Accretion Disks in Two Seyfert 1 Galaxies}

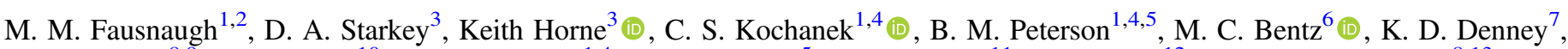

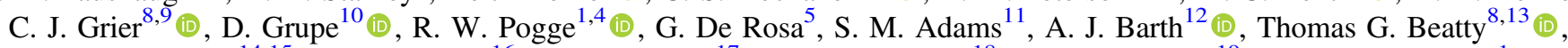
A. Bhattacharjee ${ }^{14,15}$, G. A. Borman ${ }^{16}$, T. A. Boroson ${ }^{17}$ (1) , M. C. Bottorff ${ }^{18}$, Jacob E. Brown ${ }^{19}$, Jonathan S. Brown ${ }^{1}$ (1),

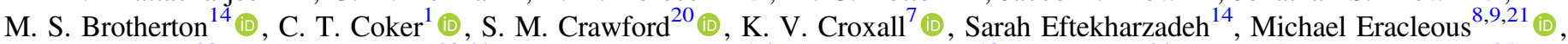
M. D. Joner ${ }^{22}$, C. B. Henderson ${ }^{23,44}$ (10, T. W.-S. Holoien ${ }^{1,4}$ (10, T. Hutchison ${ }^{18}$, Shai Kaspi ${ }^{24}$, S. Kim ${ }^{1}$, Anthea L. King ${ }^{25}$, Miao $\mathrm{Li}^{26}$ (1), Cassandra Lochhaas ${ }^{1}$, Zhiyuan Ma ${ }^{19}$ (1), F. MacInnis ${ }^{18}$, E. R. Manne-Nicholas ${ }^{5}$, M. Mason ${ }^{14}$, Carmen Montuori ${ }^{27}$, Ana Mosquera $^{28}$, Dale Mudd ${ }^{1}$ (1), R. Musso ${ }^{18}$, S. V. Nazarov ${ }^{16}$, M. L. Nguyen ${ }^{14}$, D. N. Okhmat ${ }^{16}$, Christopher A. Onken ${ }^{29}$ (1), B. Ou-Yang ${ }^{6}$, A. Pancoast ${ }^{30,45}$ (10) L. Pei ${ }^{12,31}$, Matthew T. Penny ${ }^{1,46}{ }^{(10}$, Radosław Poleski ${ }^{1}$, Stephen Rafter ${ }^{32}$, E. Romero-Colmenero ${ }^{19,33}$, Jessie Runnoe ${ }^{8,9,34}$, David J. Sand ${ }^{35}{ }^{(\mathbb{D}}$, Jaderson S. Schimoia ${ }^{36}{ }^{1}$, S. G. Sergeev ${ }^{16}$, B. J. Shappee ${ }^{37,47,48}$ (1) , Gregory V. Simonian ${ }^{1}$, Garrett Somers ${ }^{38,49}$ (i), M. Spencer ${ }^{22}$ (i), Daniel J. Stevens ${ }^{1}$, Jamie Tayar $^{1}$ (i),

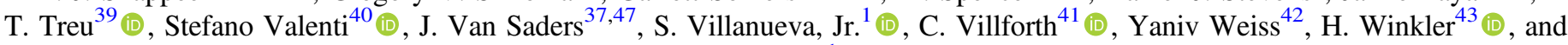
W. Zhu ${ }^{1}$

${ }^{1}$ Department of Astronomy, The Ohio State University, $140 \mathrm{~W}$ 18th Ave, Columbus, OH 43210, USA

${ }^{2}$ MIT Kavli Institute for Space and Astrophysics Research, 77 Massachusetts Avenue, 37-241, Cambridge, MA 02139, USA ${ }^{3}$ SUPA Physics and Astronomy, University of St. Andrews, Fife, KY16 9SS Scotland, UK

${ }^{4}$ Center for Cosmology and AstroParticle Physics, The Ohio State University, 191 West Woodruff Ave, Columbus, OH 43210, USA

${ }^{5}$ Space Telescope Science Institute, 3700 San Martin Drive, Baltimore, MD 21218, USA

${ }^{6}$ Department of Physics and Astronomy, Georgia State University, Atlanta, GA 30303, USA Illumination Works LLC., 5650 Blazer Parkway, Suite 152 Dublin, OH 43017, USA

${ }^{8}$ Department of Astronomy \& Astrophysics, The Pennsylvania State University, 525 Davey Laboratory, University Park, PA 16802, USA

${ }^{9}$ Institute for Gravitation and the Cosmos, The Pennsylvania State University, University Park, PA 16802, USA

${ }^{10}$ Department of Earth and Space Sciences, Morehead State University, Morehead, KY, USA

${ }^{11}$ Cahill Center for Astrophysics, California Institute of Technology, Pasadena, CA 91125, USA

${ }^{12}$ Department of Physics and Astronomy, 4129 Frederick Reines Hall, University of California, Irvine, CA 92697, USA

${ }^{13}$ Center for Exoplanets and Habitable Worlds, The Pennsylvania State University, 525 Davey Lab, University Park, PA 16802, USA

${ }^{14}$ Department of Physics and Astronomy, University of Wyoming, 1000 E. University Ave. Laramie, WY, USA

${ }^{15}$ The Department of Biology, Geology, and Physical Sciences, Sul Ross State University, WSB 216, Box-64, Alpine, TX 79832, USA

${ }^{16}$ Crimean Astrophysical Observatory, P/O Nauchny, Crimea' 298409, Russia

${ }^{17}$ Las Cumbres Global Telescope Network, 6740 Cortona Drive, Suite 102, Santa Barbara, CA 93117, USA

${ }^{18}$ Fountainwood Observatory, Department of Physics FJS 149, Southwestern University, 1011 E. University Ave., Georgetown, TX 78626, USA ${ }^{19}$ Department of Physics and Astronomy, University of Missouri, Columbia, USA

${ }^{20}$ South African Astronomical Observatory, P.O. Box 9, Observatory 7935, Cape Town, South Africa

${ }^{21}$ Center for Relativistic Astrophysics, Georgia Institute of Technology, Atlanta, GA 30332, USA

${ }^{22}$ Department of Physics and Astronomy, N283 ESC, Brigham Young University, Provo, UT 84602-4360, USA

${ }^{23}$ Jet Propulsion Laboratory, California Institute of Technology, 4800 Oak Grove Drive, Pasadena, CA 91109, USA

${ }^{24}$ School of Physics and Astronomy, Raymond and Beverly Sackler Faculty of Exact Sciences, Tel Aviv University, Tel Aviv 69978, Israel

${ }^{25}$ School of Physics, University of Melbourne, Parkville, VIC 3010, Australia

${ }^{26}$ Department of Astronomy, Columbia University, 550 W120th Street, New York, NY 10027, USA

${ }^{27}$ DiSAT, Universita dell'Insubria, via Valleggio 11, I-22100, Como, Italy

${ }^{28}$ Physics Department, United States Naval Academy, Annapolis, MD 21403, USA

${ }^{29}$ Research School of Astronomy and Astrophysics, Australian National University, Canberra, ACT 2611, Australia

${ }^{30}$ Harvard-Smithsonian Center for Astrophysics, 60 Garden Street, Cambridge, MA 02138, USA

${ }^{31}$ Department of Astronomy, University of Illinois at Urbana- Champaign, Urbana, IL 61801, USA

${ }^{32}$ Department of Physics, Faculty of Natural Sciences, University of Haifa, Haifa 31905, Israel

${ }^{33}$ Southern African Large Telescope Foundation, P.O. Box 9, Observatory 7935, Cape Town, South Africa

${ }^{34}$ Department of Astronomy, University of Michigan, 1085 S. University, 311 West Hall, Ann Arbor, MI 48109-1107, USA

${ }^{35}$ Physics \& Astronomy Department, Texas Tech University, Box 41051, Lubbock, TX 79409-1051, USA

${ }^{36}$ Instituto de Física, Universidade Federal do Rio Grande do Sul, Campus do Vale, Porto Alegre, RS, Brazil ${ }^{37}$ Carnegie Observatories, 813 Santa Barbara Street, Pasadena, CA 91101, USA

${ }^{38}$ Department of Physics and Astronomy, Vanderbilt University, 6301 Stevenson Circle, Nashville, TN 37235, USA

Department of Physics and Astronomy, University of California, Los Angeles, CA 90095-1547, USA

${ }^{40}$ Department of Physics, University of California, One Shields Avenue, Davis, CA 95616, USA

${ }^{41}$ University of Bath, Department of Physics, Claverton Down, BA2 7AY, Bath, UK

${ }^{42}$ Physics Department, Technion, Haifa 32000, Israel

${ }^{43}$ Department of Physics, University of Johannesburg, P.O. Box 524, 2006 Auckland Park, South Africa

Received 2017 March 24; revised 2017 December 15; accepted 2017 December 24; published 2018 February 16

\footnotetext{
${ }^{44}$ NASA Postdoctoral Program Fellow.

${ }^{45}$ Einstein Fellow.

46 Sagan Fellow.

47 Carnegie-Princeton Fellow.

48 Hubble Fellow.

49 VIDA Postdoctoral Fellow.

$\dagger$ While the AAS journals adhere to and respect UN resolutions regarding the designations of territories (available at http://www.un.org/press/en), it is our policy to use the affiliations provided by our authors on published articles.
} 


\begin{abstract}
We present optical continuum lags for two Seyfert 1 galaxies, MCG+08-11-011 and NGC 2617, using monitoring data from a reverberation mapping campaign carried out in 2014. Our light curves span the ugriz filters over four months, with median cadences of 1.0 and 0.6 days for MCG+08-11-011 and NGC 2617, respectively, combined with roughly daily X-ray and near-UV data from Swift for NGC 2617. We find lags consistent with geometrically thin accretion-disk models that predict a lag-wavelength relation of $\tau \propto \lambda^{4 / 3}$. However, the observed lags are larger than predictions based on standard thin-disk theory by factors of 3.3 for MCG+08-11-011 and 2.3 for NGC 2617. These differences can be explained if the mass accretion rates are larger than inferred from the optical luminosity by a factor of 4.3 in MCG+08-11-011 and a factor of 1.3 in NGC 2617, although uncertainty in the SMBH masses determines the significance of this result. While the X-ray variability in NGC 2617 precedes the $\mathrm{UV} /$ optical variability, the long (2.6 day) lag is problematic for coronal reprocessing models.
\end{abstract}

Key words: accretion, accretion disks - galaxies: individual (MCG+08-11-011, NGC 2617) - galaxies: Seyfert

Supporting material: machine-readable tables

\section{Introduction}

Energy generation in active galactic nuclei (AGNs) is believed to be due to an accretion disk around a supermassive black hole (SMBH). Viscous torques in the disk caused by magnetic fields move matter closer to the SMBH and convert gravitational potential energy into heat and radiation (e.g., Page \& Thorne 1974; Rees 1984; Balbus \& Hawley 1998). The disk reaches $10^{5}-10^{6} \mathrm{~K}$ at its inner edge, with a gradient to cooler temperatures at larger radii, leading to a continuum emission spectrum spanning the extreme ultraviolet (UV) to the infrared (IR). This model is sufficient to explain the large luminosities and UV peaks of typical AGN spectral energy distributions (Burbidge 1967; Weedman 1977; Shields 1978; Elvis et al. 1994; Telfer et al. 2002). The UV/optical continuum from the disk also provides the seed photons that are reprocessed into the IR by hot dust (e.g., Suganuma et al. 2006; Nenkova et al. 2008; Vazquez et al. 2015) and X-rays by a putative hot "corona" (e.g., Haardt \& Maraschi 1991; Reynolds \& Nowak 2003; Turner et al. 2006). The hottest parts of the disk supply the ionizing photons that power Doppler-broadened emission lines in the broad- and narrowline regions (BLRs and NLRs, Davidson \& Netzer 1979; Veilleux \& Osterbrock 1987).

Many important aspects of the accretion disk and the continuum emission remain unknown. On the theoretical side, there are several viable accretion-disk models, ranging from geometrically thin disks that radiate thermally (Shakura \& Sunyaev 1973) to thick toroids that radiate through plasma processes (Abramowicz et al. 1988), as well as radiatively inefficient accretion flows that advect most of their energy across the event horizon (Narayan \& Yi 1995). It is also challenging to account for the $\mathrm{X}$-ray-emitting corona from first principles (Schnittman et al. 2013), and there are a wide variety of hypothesized geometries and energetic connections between the corona and the accretion disk. Simulations have recently made progress by incorporating radiation transport (e.g., Schnittman \& Krolik 2013; Sạdowski et al. 2014; Sạdowski \& Narayan 2015), but it has not been possible to simulate the full magneto-radiation-hydrodynamics of the disk that are fundamental for determining its observational appearance (Blaes 2014). It is also observationally difficult to isolate the intrinsic disk continuum due to line emission, host-galaxy starlight, and internal reddening, and the emission peak is generally unobservable due to absorption by intervening hydrogen. After accounting for these effects, it is sometimes possible to fit the observed spectrum with disk models (e.g., Capellupo et al. 2015), but this is not always the case (e.g., Shankar et al. 2016). Alternative attempts to isolate the continuum emission have made use of polarimetry (Kishimoto et al. 2004, 2008) and difference spectra/color variability (Wilhite et al. 2005; Pereyra et al. 2006; Schmidt et al. 2012), but the interpretation of these data is not straightforward (e.g., Kokubo 2015, 2016).

Reverberation mapping (RM, Blandford \& McKee 1982; Peterson 1993, 2014) is a powerful tool for the investigation of AGN accretion disks. The basic principle of RM is to search for temporal correlations between the time-variable emission at different wavelengths, which encode information about unresolved structures in the AGN. The formalism for RM is a convolution operation

$$
f_{R}(t)=\int f_{S}(t-\tau) \Psi(\tau) d \tau,
$$

where $f_{S}$ is the driving signal light curve, $f_{R}$ is the reverberating light curve, $\tau$ is the time delay (or "lag"), and $\Psi(\tau)$ is the transfer function. The transfer function is determined by the matter distribution surrounding the source of $f_{S}$, and the main goal of RM is to infer $\Psi(\tau)$ from observations of $f_{S}$ and $f_{R}$. Recovering the transfer function is an ill-posed inverse problem that requires regularization (Horne 1994; Skielboe et al. 2015) or forward modeling (Pancoast et al. 2014; Starkey et al. 2016) to solve. However, one can still infer a great deal of information from the cross-correlation of $f_{S}$ and $f_{R}$ to determine the mean lag $\langle\tau\rangle$ between the light curves, which is related to the first moment of $\Psi(\tau)$. Combined with the speed of light, the lag determines the characteristic size of the reverberating structure.

In the context of the accretion disk, reverberation signals are expected because of reprocessing arguments: shorter-wavelength emission originates near the black hole where the disk is hottest, while longer-wavelength emission originates in the cooler parts of the disk at larger radii. Self-irradiation by shortwavelength emission deposits energy in the outer part of the disk, contributing an extra heating term (Shakura \& Sunyaev 1973). As the short-wavelength emission varies, it drives variations at longer wavelengths delayed by the light travel time across the disk. This model predicts that shortwavelength variations will lead long-wavelength variations after a time delay that scales with the size of the disk (e.g., Krolik et al. 1991).

Measuring these interband continuum lags is extremely difficult because the predicted size of the accretion disk is only 
Table 1

Physical Parameters

\begin{tabular}{|c|c|c|c|c|c|}
\hline $\begin{array}{l}\text { Object } \\
\text { (1) }\end{array}$ & $\begin{array}{c}\text { Redshift } \\
z \\
(2)\end{array}$ & $\begin{array}{c}\text { Mass } \\
M_{\mathrm{BH}}\left(M_{\odot}\right) \\
(3)\end{array}$ & $\begin{array}{l}\text { Luminosity } \\
L\left(\mathrm{erg} \mathrm{s}^{-1}\right) \\
\text { (4) }\end{array}$ & $\begin{array}{c}\text { Eddington Ratio } \\
\dot{m}_{\mathrm{Edd}} \\
(5)\end{array}$ & $\begin{array}{c}\text { Accretion Rate } \\
\left(M_{\odot} \mathrm{yr}^{-1}\right) \\
(6)\end{array}$ \\
\hline$\overline{\mathrm{MCG}+08-11-011}$ & 0.0205 & $\left(2.82_{-1.86}^{+5.50}\right) \times 10^{7}$ & $(1.98 \pm 0.20) \times 10^{44}$ & 0.054 & $3.5 \times 10^{-2}$ \\
\hline
\end{tabular}

Note. Columns 2, 3, and 4 are taken from Fausnaugh et al. (2017). Column 3 was derived from continuum-H $\beta$ lags, and includes all systematic uncertainties. Column 4 is calculated from the observed mean optical luminosity, assuming a bolometric correction of $10\left(L=10 \lambda L_{5100 \AA}\right)$. Note that this value has been corrected for Galactic extinction and host-galaxy starlight. Columns 6 was calculated assuming a radiative efficiency of $\eta=0.1$ (see Section 2 and Equation (3)).

about one light day (about 170 gravitational radii for a $10^{8} M_{\odot}$ black hole), and monitoring campaigns require comparable or better cadence to resolve such a short lag. The first suggestive $(2-3 \sigma)$ report was by Collier et al. (1998), who found that the UV variations led the optical variations in NGC 7469. Since then, there have been many hints of longer lags at longer wavelengths in other AGN, but most of these measurements are not statistically significant $(\leqslant 2 \sigma)$ and represent upper limits (Sergeev et al. 2005; Arévalo et al. 2008; Breedt et al. 2010; Lira et al. 2015; Troyer et al. 2016; Buisson et al. 2017; Gliozzi et al. 2017; Jiang et al. 2017). The relationship between the $\mathrm{X}$-ray and optical emission also appears to be complex and does not necessarily fit into a simple reprocessing model. In some cases, there is not enough energy in the X-ray variations to drive the long term trends in the optical light curves (Uttley et al. 2003; Arévalo et al. 2009; Breedt et al. 2009). There are also reports of optical emission leading the X-rays (Marshall et al. 2008), as well as uncorrelated X-ray/optical emission (Maoz et al. 2002).

The first secure detection $(>3 \sigma)$ of interband continuum lags was in NGC 2617 by Shappee et al. (2014), who found longer lags at longer wavelengths that were consistent with predictions for reprocessing in a standard geometrically thin accretion disk. The only other AGN with significant continuum lag detections is NGC 5548. McHardy et al. (2014) resolved continuum lags in this object using two years of Swift data, while the Space Telescope and Optical Reverberation Mapping project (AGN STORM, De Rosa et al. 2015) obtained the most complete RM measurement of the accretion disk to date. The STORM project detected interband lags between the X-ray, UV, optical, and near-IR wavelengths using four space-based observatories and 25 ground-based telescopes (Edelson et al. 2015; Fausnaugh et al. 2016; Starkey et al. 2017), and the measured lagwavelength relation is again consistent with predictions for reprocessing in a geometrically thin disk. However, the size of the disk indicated by the STORM measurements is larger by a factor of three than the predictions from standard models.

This finding is consistent with results from gravitational microlensing of strongly lensed quasars. Microlensing is one of the only other ways of investigating physical scales close to the $\mathrm{SMBH}$, and studies using this method also find disk sizes larger than thin-disk theory by a factor of a few (Morgan et al. 2010; Blackburne et al. 2011; Mosquera et al. 2013; Jiménez-Vicente et al. 2014). However, microlensing can only probe the disks in distant, high-luminosity quasars, while RM provides a means of probing accretion disks in local, low-luminosity AGN. It is therefore imperative to expand the sample of objects with secure interband continuum lags, and several such programs have been completed, with others still in progress (e.g.,
NGC 4151, Edelson et al. 2017; NGC 4593, McHardy et al. 2016).

In this study, we present detections of near-UV and optical interband continuum lags in two Seyfert 1 galaxies, MCG +08 11-011 and NGC 2617. These objects were observed as part of a monitoring campaign in 2014, the original goal being to measure SMBH masses using continuum- $\mathrm{H} \beta$ reverberations. Fausnaugh et al. (2017) presented the spectroscopic monitoring component and the initial results for the broad line lags and SMBH masses. Here, we analyze four months of densely sampled (0.6-1.5 day cadence) broadband photometric monitoring data for these objects and measure the interband continuum lags. MCG+08-11-011 is a new addition to the sample of objects with secure accretion disk RM measurements. NGC 2617 is in a lower luminosity state than when observed by Shappee et al. (2014), and provides an interesting reference point for investigating the dependence of accretion disk structure on luminosity.

In Section 2, we discuss our observations, data reduction, and light curves. In Section 3, we describe our time series analysis and present interband continuum lags. In Section 4, we report results from a physical model of our data using the Continuum REprocessed AGN Markov Chain Monte Carlo code (CREAM, Starkey et al. 2016). In Section 5, we discuss our results in the context of an accretion-disk reprocessing model, and we summarize our findings in Section 6. We assume a consensus cosmology with $H_{0}=70 \mathrm{~km} \mathrm{~s}^{-1} \mathrm{Mpc}^{-1}, \Omega_{\mathrm{m}}=0.3$, and $\Omega_{\Lambda}=0.7$.

\section{Targets and Observations}

The RM campaign extended between 2014 January and July, targeting $11 \mathrm{AGN}$, and was primarily based on observations at the MDM observatory (see Fausnaugh et al. 2017 for details). Supplemental data were contributed by telescopes from around the globe, with the unique addition-in contrast to typical RM campaigns - of broadband imaging data in multiple filters. MCG+08-11-011 and NGC 2617 were the two most variable AGN during this campaign, and yielded robust measurements of the continuum- $\mathrm{H} \beta$ lags used to measure the SMBH masses (Fausnaugh et al. 2017). Because of the strong variability signals, we focused on these two objects for our first analysis of the broadband imaging data.

Table 1 presents some of the important physical parameters of these AGN. The SMBH mass $M_{\mathrm{BH}}$ was determined by Fausnaugh et al. (2017) using $5100 \AA$ continuum-H $\beta$ lags. The luminosity $L$ was derived from the average values of the $5100 \AA$ continuum light curves of the same RM campaign, assuming a bolometric correction of 10 (we discuss alternative bolometric corrections in Section 4 below). Note that these estimates are corrected for Galactic extinction and host-galaxy 
starlight (see Fausnaugh et al. 2017 for details). Defining the Eddington luminosity and accretion rate as

$$
L_{\mathrm{Edd}}=\frac{4 \pi G M_{\mathrm{BH}} c}{\kappa}
$$

and

$$
\dot{M}_{\mathrm{Edd}}=\frac{L_{\mathrm{Edd}}}{\eta c^{2}}
$$

where $\kappa$ is the Thomson opacity $\left(\sim 0.4 \mathrm{~cm}^{2} \mathrm{~g}^{-1}\right)$ and $\eta$ is the radiative efficiency, we calculate the Eddington ratio $\dot{m}_{\mathrm{Edd}}=\dot{M} / \dot{M}_{\mathrm{Edd}}=L / L_{\mathrm{Edd}}$ and mass accretion rate $\dot{M}$, setting $\eta=0.1$.

NGC 2617 was observed with Swift during this time as part of a continuing target-of-opportunity program (Shappee et al. 2014). Daily exposures were taken with all six filters of the UltraViolet/Optical Telescope (UVOT, Roming et al. 2005) with simultaneous monitoring from the X-ray Telescope (XRT, Burrows et al. 2005).

Our ground-based imaging is mostly from the Las Cumbres Observatory (LCO) $1 \mathrm{~m}$ global telescope network (Brown et al. 2013), acquired as part of the AGN Key project (Valenti et al. 2015). The network consist of nine identical one-meter telescopes distributed at four sites around the world. Each telescope has the same optical design and detectors. At the time of the RM campaign, the detectors were SBIGSTX-16803 cameras with a field of view of $16^{\prime} \times 16^{\prime}$ and a pixel scale of 0 ". 23. Data were obtained between 2014 January and May for MCG+08-11-011 and NGC 2617 in the ugriz bands on an approximately daily cadence. These filters have central wavelengths of $3600 \AA, 4800 \AA, 6300 \AA, \quad 7700 \AA$, and $9100 \AA$, respectively (see Tables 6 and 7 for corrections to the rest-frame). MCG+08-11-011 has a high declination $\left(+46^{\circ} .5\right)$ and can only be observed from LCO's northern-most site at McDonald Observatory. NGC 2617 was observed from the sites at McDonald, Siding Spring, Australia, and Sutherland, South Africa.

Both objects were also observed with the $0.7 \mathrm{~m}$ telescope at the Crimean Astrophysical Observatory (CrAO). Images were taken in the Johnson $B V R I$ bands with central wavelengths of $4400,5500,7000$, and $8800 \AA$, as well as a filter approximating the Cousins I-band, designated $R I$, which has a central wavelength of $7900 \AA$, but is much narrower than the Johnson I-band. The median cadence of these observations is about two days. The telescope is equipped with a AP7p CCD detector that has a $15^{\prime} \times 15^{\prime}$ field-of-view and pixel scale of 1 '!76. Finally, $V$-band images were obtained with the $0.9 \mathrm{~m}$ telescope at West Mountain Observatory and the $0.5 \mathrm{~m}$ telescope at Wise Observatory (Brosch et al. 2008).

The spectroscopic monitoring observations were obtained at the MDM observatory using the Boller \& Chivens CCD spectrograph on the $1.3 \mathrm{~m}$ McGraw-Hill telescope. We extracted light curves for the rest-frame $5100 \AA$ continuum, a region of the spectrum relatively free of line emission. The $2.3 \mathrm{~m}$ telescope at Wyoming Infrared Observatory (WIRO) contributed four epochs of optical spectroscopy to help fill anticipated gaps in the MDM observations. These data and our intercalibration procedures are described in detail by Fausnaugh et al. (2017).

\subsection{Image Subtraction}

We analyzed the ground-based imaging data using the image subtraction package IS IS (Alard \& Lupton 1998). Although a common reduction procedure was applied to all images, we analyzed the data sets from each telescope and in each filter separately, including the individual $1 \mathrm{~m}$ telescopes in the LCO network.

First, the raw images were bias-subtracted and flat-fielded at the contributing facility using the appropriate software reduction pipelines. Next, all images were collected in a central repository and vetted by eye for poor observing conditions or errors in the initial image processing. For each telescope, we then registered the images to a common coordinate system, and we constructed a high-quality reference image by combining the epochs with the best seeing and lowest backgrounds. Finally, we subtracted the reference from each epoch using ISIS. ISIS transforms the point-spread-function (PSF) and flux scale of one image to match that of a second image by fitting for a spatially variable convolution kernel. The subtraction leaves a clean measurement of the variable flux on a pixel-by-pixel basis.

\subsection{Light Curves}

The Swift UVOT light curves were extracted using standard aperture photometry techniques. We used a 5!"0 radius circular aperture centered on the AGN using the UVOT software task uvotsource. Background counts were estimated using the mode of pixel values in a surrounding annulus 15 !" 0 in width. The large annulus was chosen to sample the background sky level, so this procedure introduces a constant level of contamination from the host-galaxy starlight within the 5!"0 aperturehowever, the contamination does not affect our final results, which only depend on the differential variations of the light curves. The XRT data were reduced with the xrtpipeline task included in the HEASOFT package, using the same apertures, response files, and modeling techniques described in Shappee et al. (2014).

For the ground-based data, we extracted differential light curves from the subtracted images using the photometry package included with ISIS. First, the software fits a model to the reference image PSF. Then, for all sources identified in the reference image, the software smooths the model PSF by the convolution kernel fit during the image subtraction and uses the result to perform PSF photometry on the subtracted image. The result is a light curve in units of differential counts relative to the flux of the object in the reference image. These flux variations are free of constant contaminates, such as hostgalaxy starlight, and extrinsic variations due to seeing or aperture effects.

ISIS accounts for only the local Poisson uncertainty on the observed counts. To account for any systematic issues associated with the image subtraction, we rescaled the light curve uncertainties to match the residuals of comparison stars in the field-of-view. Our method closely follows that of Fausnaugh et al. (2016). For each epoch, we compared the differential flux of each star to ISIS's estimate of its uncertainty by calculating the rescaling factor required to make the flux residuals consistent with zero at $1 \sigma$. We then rescaled the flux uncertainties at that epoch by the median of the rescaling factors of all comparison stars. We imposed a minimum rescaling factor of one, because the photon noise sets 
a fundamental floor on the precision, and we removed obvious variable stars by censoring light curves with long-term trends or mean rescaling factors greater than 100. On average, this forces the comparison star light curves to have a reduced $\chi^{2}$ of 1 for a constant model. We generally found rescaling factors ranging between 1.0 and 6.0 , depending on the quality of the data and the number of comparison stars. One LCO telescope at Siding Spring had rescaling factors that reached 10 and 12 in the $u$-band and $g$-band, while one LCO telescope at Sutherland had rescaling factors between 5 and 8 for all bands. These telescopes had fewer observations overall, limiting our ability to construct a good reference image, and the image subtraction quality suffers as a result.

To combine light curves from different telescopes, we used the intercalibration procedure described by Fausnaugh et al. (2016). Briefly, the calibration solves for maximum likelihood offsets and rescaling factors, which account for the different flux levels in the reference images of each telescope and the different definitions of counts (due to heterogeneous detector responses, filter throughputs, gains, etc.). To first order, the linear calibration model also accounts for slight variations in the effective wavelengths and widths of the different filter passbands. Due to the limited amount of CrAO data, we combined the $B$-band with the $g$-band, the $R$-band with the $r$-band, the $R I$ band with the $i$-band, and the $I$-band with the $z$-band. The CrAO $V$-band light curve was incorporated with the spectroscopic continuum light curve from MDM.

Because observations at different telescopes are never taken simultaneously, it is necessary to interpolate the light curves when solving for the calibration shifts and rescaling factors. We predicted the light curve values at intermediate times using JAVELIN ( $\mathrm{Zu}$ et al. 2011), which models the light curves with a stochastic process model. Process models with different covariances/power spectra are available with JAVELIN, but we have found that the damped-random walk (DRW, or Ornstein-Uhlenbeck process) is adequate for this purpose. As discussed in Fausnaugh et al. (2017), our light curves are not long enough to constrain the damping timescale of the process model, so we fixed this parameter to 200 days (see also Kozłowski 2017).

Finally, we flux-calibrated the differential light curves by performing aperture photometry on the reference image of one standardized data set. We chose the McDonald LCO data as the standard, because this light curve has the largest number of observations. All other light curves are transformed to match the flux scale and mean value of this light curve using the JAVELIN intercalibration routine. Flux calibration then reduces to measuring the reference image's zero-point magnitude and the total counts of the AGN. We used a 5!"0 radius circular aperture and a sky annulus of 15 !. 0 . We did the same for all comparison stars, and measured $\mathrm{AB}$ magnitude zero points in each image by matching to the SDSS DR7 photometric catalog (Abazajian et al. 2009). The final flux measurements are again contaminated by the host-galaxy starlight in the reference image, though this contamination does not contribute to the variations measured from the image subtraction. See Fausnaugh et al. (2016) for a more thorough discussion of this flux-calibration technique.

The light curves are given in Tables 2 through 4, and shown in the left-hand panels of Figures 1-3. Table 5 summarizes useful properties of the light curves. For MCG+08-11-011, the median cadence is about 1 day ( 1.5 days in the $u$-band), and for
Table 2

MCG+08-11-011 Light Curves

\begin{tabular}{|c|c|c|c|}
\hline Filter & $\begin{array}{l}\text { HJD-2,400,000 } \\
\text { (days) }\end{array}$ & $\begin{array}{c}F_{\lambda} \\
\left(10^{-15} \mathrm{erg} \mathrm{cm}^{-2} \mathrm{~s}^{-1} \AA^{-1}\right)\end{array}$ & Telescope ID \\
\hline$u$ & 56683.5826 & $4.9925 \pm 0.1199$ & LCO1 \\
\hline$u$ & 56684.5841 & $4.9352 \pm 0.1691$ & LCO1 \\
\hline$u$ & 56687.5629 & $5.5462 \pm 0.1651$ & $\mathrm{LCO} 1$ \\
\hline$\cdots$ & $\cdots$ & $\cdots$ & $\cdots$ \\
\hline$g$ & 56639.5200 & $6.4602 \pm 0.0266$ & $\mathrm{CrAO}$ \\
\hline$g$ & 56649.4759 & $6.8878 \pm 0.1825$ & $\mathrm{CrAO}$ \\
\hline$g$ & 56653.4950 & $6.6852 \pm 0.0309$ & $\mathrm{CrAO}$ \\
\hline$\cdots$ & $\cdots$ & $\cdots$ & $\cdots$ \\
\hline$r$ & 56682.5935 & $8.1803 \pm 0.0420$ & $\mathrm{LCO} 1$ \\
\hline$r$ & 56683.5838 & $8.4089 \pm 0.0454$ & LCO1 \\
\hline$r$ & 56684.5854 & $8.4846 \pm 0.0546$ & LCO1 \\
\hline$\cdots$ & $\cdots$ & $\cdots$ & $\cdots$ \\
\hline$i$ & 56682.5940 & $6.4003 \pm 0.0316$ & LCO1 \\
\hline$i$ & 56683.5992 & $6.3211 \pm 0.0344$ & LCO1 \\
\hline$i$ & 56684.5858 & $6.5392 \pm 0.0365$ & LCO1 \\
\hline$\cdots$ & $\cdots$ & $\cdots$ & $\cdots$ \\
\hline$z$ & 56682.5945 & $5.7181 \pm 0.0297$ & $\mathrm{LCO} 1$ \\
\hline$z$ & 56683.5997 & $5.7453 \pm 0.0306$ & LCO1 \\
\hline$z$ & 56684.5863 & $5.7661 \pm 0.0318$ & LCO1 \\
\hline$\cdots$ & $\cdots$ & .. & $\cdots$ \\
\hline
\end{tabular}

(This table is available in its entirety in machine-readable form.)

NGC 2617, the median cadence is about 0.6 days in the ugriz bands and 1.1-1.2 days for the Swift data. We also selfconsistently estimated the mean flux $\hat{F}$ and the intrinsic variability $\sigma_{\mathrm{var}}$ (corrected for measurement noise) by solving for the values of these parameters that minimize

$$
-2 \ln \mathcal{L}=\sum_{i}^{N_{t}} \frac{\left[F\left(t_{i}\right)-\hat{F}\right]^{2}}{\sigma^{2}\left(t_{i}\right)+\sigma_{\mathrm{var}}^{2}}+\sum_{i}^{N_{t}} \ln \left[\sigma^{2}\left(t_{i}\right)+\sigma_{\mathrm{var}}^{2}\right],
$$

where $F\left(t_{i}\right)$ is the flux measurement at time $t_{i}$ and $\left.\sigma_{(} t_{i}\right)$ is its uncertainty. This procedure is identical to that of Fausnaugh et al. (2017), and we similarly report the rms fractional variability amplitude $F_{\text {var }}=\sigma_{\text {var }} / \hat{F}$, the mean signal-to-noise $\langle\mathrm{S} / \mathrm{N}\rangle$, and the signal-to-noise of $F_{\text {var }}$

$$
(\mathrm{S} / \mathrm{N})_{\mathrm{var}}=\frac{\sigma_{\mathrm{var}}}{\bar{\sigma} \sqrt{2 / N_{\mathrm{obs}}}}
$$

for each light curve, where $\bar{\sigma}$ is the mean measurement uncertainty among observations and $N_{\mathrm{obs}}$ is the number of observations. The right-hand side of Equation (5) is derived by assuming that the variance of $\bar{\sigma}$ has a reduced $\chi^{2}$ distribution.

\section{Time Series Analysis}

We searched for lags in the continuum light curves using the interpolated cross-correlation function (ICCF) and the Bayesian model of JAVELIN. Full descriptions of the cross-correlation technique can be found in Gaskell \& Peterson (1987), White \& Peterson (1994), and Peterson et al. (2004), while a complete description of JAVELIN can be found in $\mathrm{Zu}$ et al. (2011, 2013).

In brief, the ICCF method uses piecewise linear interpolation to estimate the cross-correlation coefficient $r_{\mathrm{cc}}$ for two light 


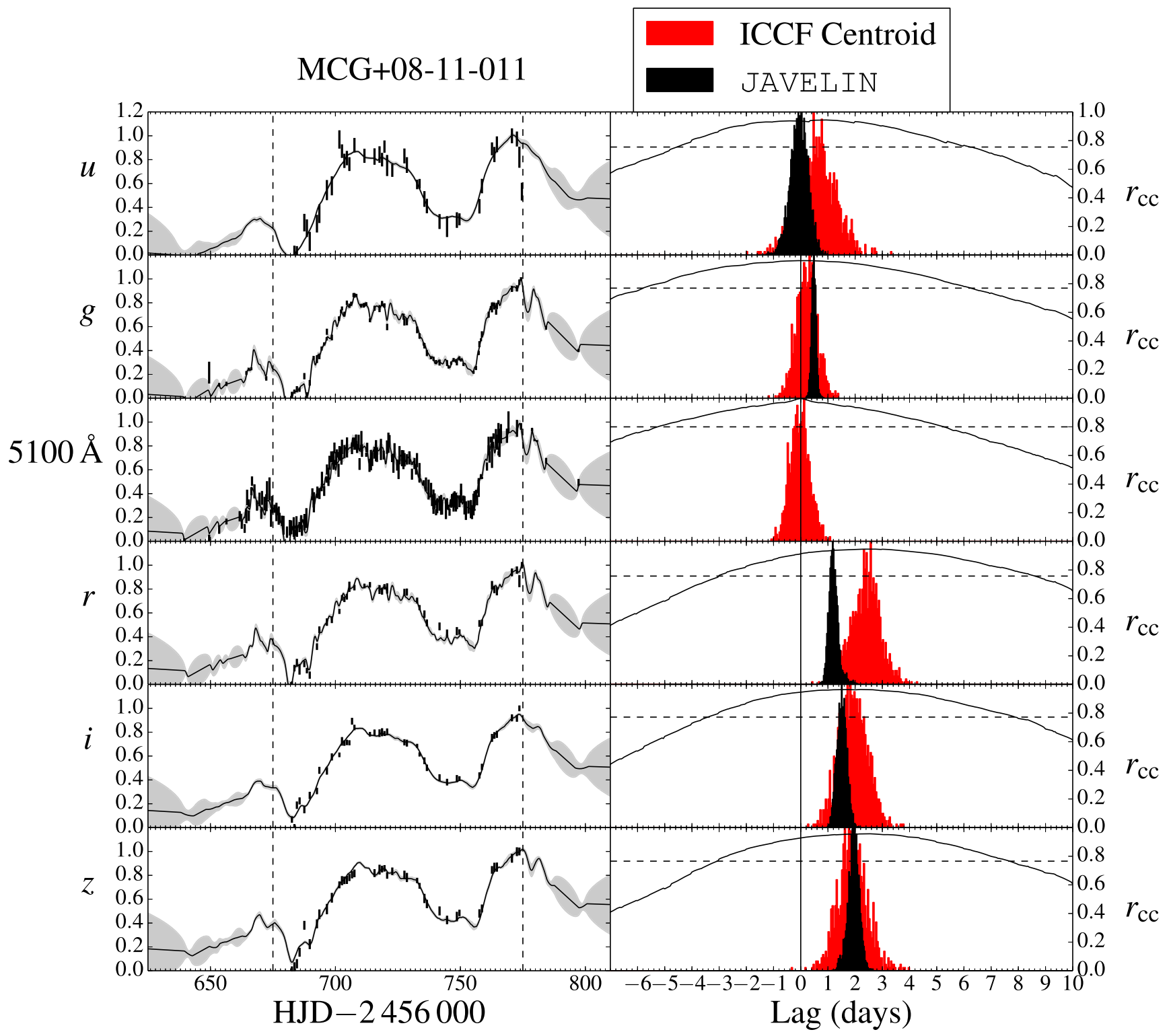

Figure 1. Left panel: light curves of MCG+08-11-011. The ugriz data are from differential broadband photometry (with $1 \sigma$ uncertainties), while the $5100 \AA$ light curve combines spectroscopy and $V$-band imaging. The $y$-axes are in flux units and scaled so that the minimum value of the light curve is 0 and the maximum value is 1. Vertical dashed lines show the restricted temporal baseline used to calculate the interpolated cross-correlation function (ICCF). The solid black lines and shaded regions show the JAVELIN interpolation and their $1 \sigma$ uncertainties. Right panel: lag estimates relative to the $5100 \AA$ continuum. The black lines show the ICCF (or the autocorrelation function for the $5100 \AA$ continuum). The horizontal dashed lines show the threshold value of correlation coefficient $r_{\mathrm{cc}}=0.8 r_{\mathrm{max}}$ used to calculated the ICCF centroid. The red histograms show the ICCF centroid distributions from the FR/RSS method (Section 3), and the black histograms show the JAVELIN posterior lag distributions.

curves after shifting one by a given lag $\tau$. We evaluated the ICCF on a grid of lags spaced by 0.05 days, and we estimated the lag between the two light curves using the ICCF centroid. The ICCF centroid $\tau_{\text {cent }}$ is defined as the average $\tau$ weighted by $r_{\mathrm{cc}}$ for $r_{c c}>0.8 r_{\max }$, where $r_{\max }$ is the maximum of the ICCF. For completeness, we also report the lag $\tau_{\text {peak }}$ that corresponds to $r_{\max }$. Uncertainties on the lag were estimated using the flux randomization/random subset sampling (FR/RSS) method of Peterson et al. (2004). Individual points from each light curve were resampled (with replacement) and adjusted by random Gaussian deviates scaled to the measurement uncertainties, and then the centroid $\tau_{\text {cent }}$ was recalculated. After repeating this procedure $10^{3}$ times, the central $68 \%$ interval of the resulting centroid distribution was adopted for the uncertainty in $\tau_{\text {cent }}$.
JAVELIN determines the lags between light curves by modeling the data as a stochastic process (a DRW) and fitting for the transfer function $\Psi(\tau)$ (see Section 1). The formalism assumes that the transfer function can be approximated by a top hat, as well as parameterized by a scaling factor, width, and central time delay. The central time delay is adopted as a measure of the lag, which we designate $\tau_{\text {JAV }}$. Skielboe et al. (2015) have shown that measurements of the lag do not depend on the choice of stochastic process used to describe light curve variations. JAVELIN can also fit multiple light curves and their underlying lags simultaneously, which maximizes the amount of information used in the fit and accounts for covariances between the lags of different light curves (note that there is no prior on the relations between the transfer functions for the different light curves). 


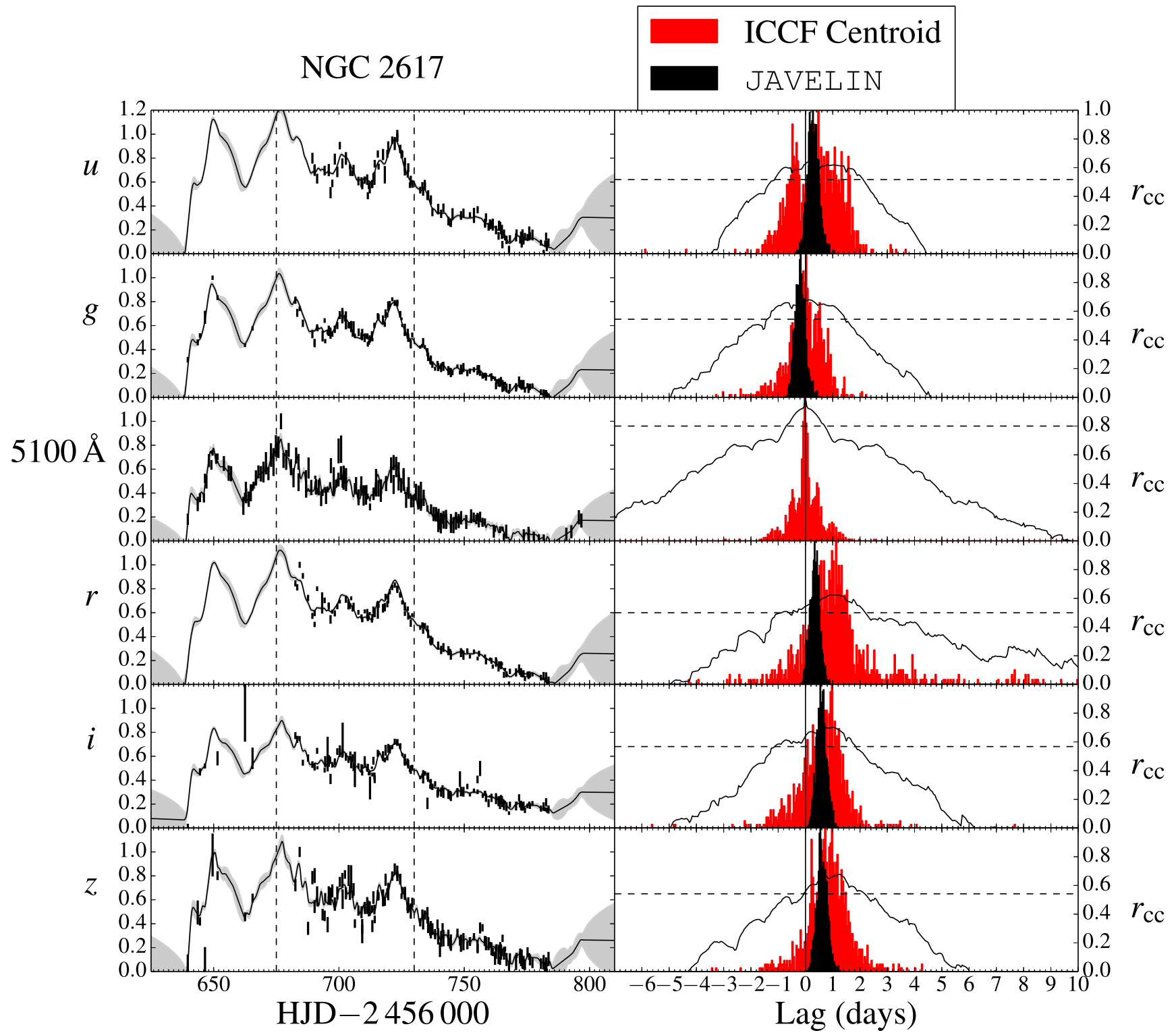

Figure 2. Same as Figure 1, but for the ground-based light curves of NGC 2617.

In each case, we measured the lags relative to the $5100 \AA$ light curve. This choice is unimportant for JAVELIN-but in the ICCF analysis, it is important to use the best light curve in terms of sampling and noise properties as the reference light curve. For the ICCF method, we shifted and interpolated both light curves, and used the average value of $r_{\mathrm{cc}}$ to estimate $\tau_{\text {cent }}$. We restricted the light curve baselines to $6675<\mathrm{HJD}-$ $2,450,000<6775$ days for MCG+08-11-011, to avoid interpolating over large gaps in the $g$-band. For NGC 2617, we restricted the baseline to $6675<\mathrm{HJD}-2,450,000<6730$ days, to avoid the gradual flux variations at the tail of the light curves. Gradual variations such as these can affect the ICCFs, due to red-noise leakage (Welsh 1999, see also Fausnaugh et al. 2017). The Swift light curves begin somewhat earlier than the ground-based data (6630 days), and we include these earlier observations in our analysis. We did not otherwise detrend the data.

For the JAVELIN models of MCG+08-11-011, we fit all of the ugriz data simultaneously. For NGC 2617, the combination of Swift and ugriz light curves was too large for JAVELIN to converge on a solution in a reasonable amount of time, so we fit the Swift and ground-based data sets separately. JAVELIN removes any linear trends from the light curves in the fits, and we did not limit the temporal baselines when fitting with JAVELIN.

\subsection{Results}

In Figures 1-3, we show the ICCFs, lag centroid distributions, and JAVELIN posterior lag distributions for each light curve. Table 6 gives the lags and their uncertainties for MCG $+08-11-011$, and Table 7 gives the same for NGC 2617, both corrected to the rest-frame. The lags derived from the ICCF and JAVELIN approaches are consistent, except for the $r$-band in MCG+08-11-011: the ICCF centroid distribution gives a restframe lag of $2.56 \pm 0.51$ days, while JAVELIN finds a lag of $1.19 \pm 0.16$ days. We found that this difference is related to interpolation of the $r$-band light curve over the large gaps in the second half of the campaign. If we only interpolate the $5100 \AA$ continuum light curve, the ICCF lag is $\tau_{\text {cent }}=2.19 \pm 0.59$ 


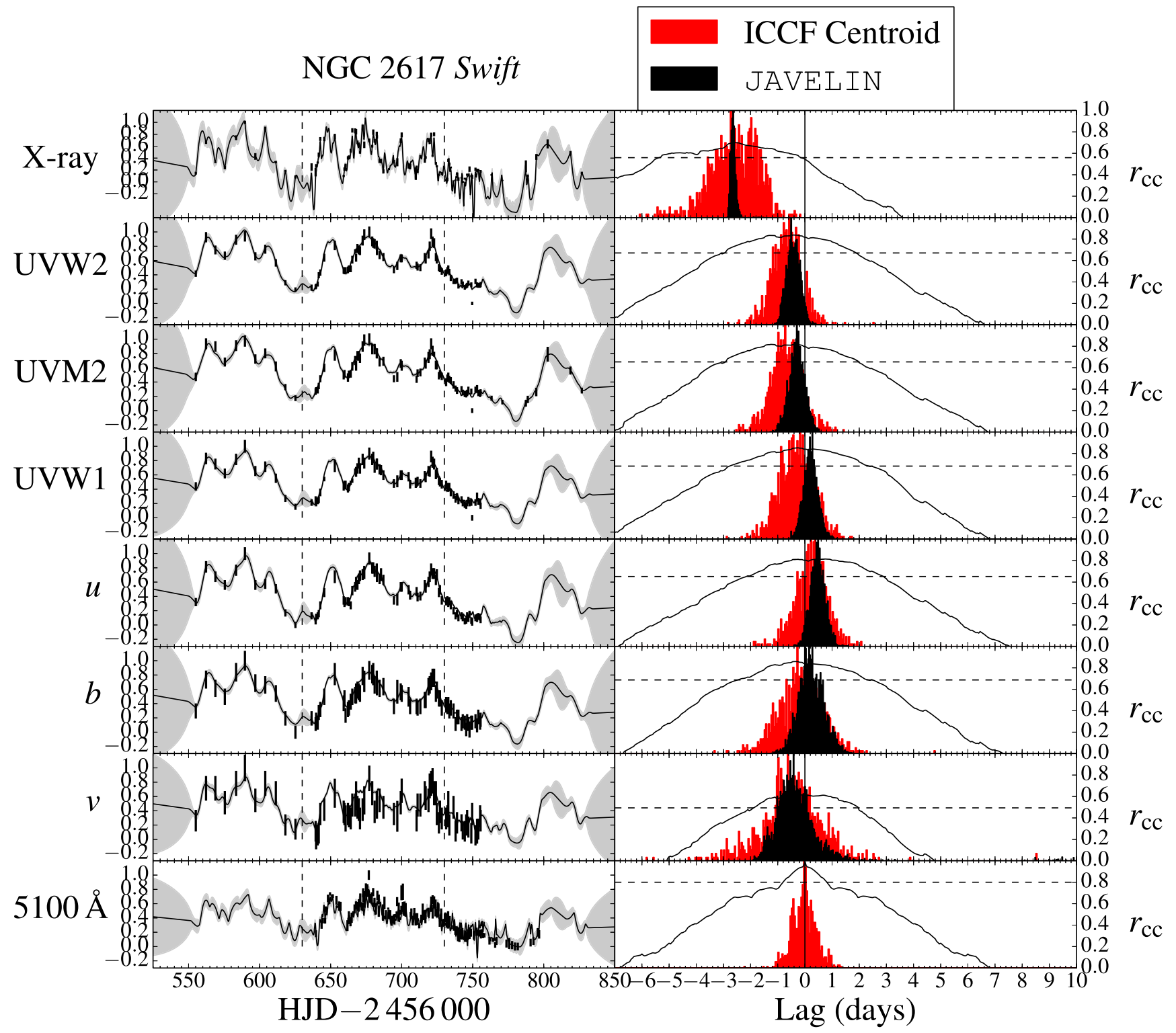

Figure 3. Same as Figure 1, but for the Swift light curves of NGC 2617.

days, which reduces the discrepancy from $2.7 \sigma$ to $1.7 \sigma$. The widths of the JAVELIN posteriors are much smaller than the ICCF centroid distributions - and for NGC 2617, we must rely on JAVELIN to claim statistically significant detections. The JAVELIN distributions are also narrower for the Swift data, although the UV-optical lags are only detected at the 0.9-1.5 $\sigma$ level, which may be related to the longer cadence of these light curves. The uncertainty in the lag from the ICCF method is intrinsically limited by the width of the autocorrelation of the continuum light curve (Peterson 1993); given that, and the better precision using JAVELIN, we adopt $\tau_{\text {JAV }}$ for our final lag measurements. JAVELIN also accounts for the correlations between lags from light curves at different wavelengths, and therefore maximizes the amount of information used in the fit. Overall, the uncertainties on the lag are unlikely to be any smaller than the estimates from JAVELIN, while the ICCF centroid distributions probably place upper limits on the lag uncertainties.

Our results are largely consistent with a disk reprocessing model, with larger lags at longer wavelengths. In fact, the trends are nearly monotonic, with the main exceptions being the $u$-band lags in NGC 2617 and the $g$-band lag in MCG+0811-011. The Swift UVW1, b, and $v$ band lags in NGC 2617are also contrary to this trend, but are consistent with 0 days at less than $1 \sigma$.

The $u$-band lags in NGC 2617 are detected at $1.5 \sigma$ and $2.0 \sigma$, respectively. These filters are contaminated by Balmer continuum emission from the BLR, which is expected to reverberate on longer timescales than the continuum emission and may bias the observed lags to larger values. This bias has been seen in NGC 5548 (Edelson et al. 2015; Fausnaugh et al. 2016) and the Swift monitoring data of NGC 2617 from 2013 analyzed by Shappee et al. (2014).

The $g$-band lag relative to $5100 \AA$ in $\mathrm{MCG}+08-11-011$ of $0.50 \pm 0.08$ days is detected at high significance $(6.25 \sigma)$. It is less clear what might be affecting this band, so we investigated the cause of the lag in more detail. One possibility is that the uncertainties on the data are underestimated. To check this, we re-ran the FR/RSS procedure and the JAVELIN fits with the $g$-band uncertainties inflated by factors of 1.5 and 3.0, because 
Table 3

NGC 2617 Light Curves

\begin{tabular}{|c|c|c|c|}
\hline Filter & $\begin{array}{l}\text { HJD—2,400,000 } \\
\text { (days) }\end{array}$ & $\begin{array}{c}F_{\lambda} \\
\left(10^{-15} \mathrm{erg} \mathrm{cm}^{-2} \mathrm{~s}^{-1} \AA^{-1}\right)\end{array}$ & Telescope ID \\
\hline$u$ & 56689.3963 & $6.9971 \pm 0.0770$ & $\mathrm{LCO5}$ \\
\hline$u$ & 56690.2849 & $6.8172 \pm 0.0719$ & LCO5 \\
\hline$u$ & 56690.2916 & $6.8395 \pm 0.0701$ & $\mathrm{LCO} 4$ \\
\hline$\cdots$ & $\cdots$ & $\cdots$ & $\cdots$ \\
\hline$g$ & 56639.6731 & $6.3221 \pm 0.2172$ & $\mathrm{CrAO}$ \\
\hline$g$ & 56643.6272 & $7.6720 \pm 0.2321$ & $\mathrm{CrAO}$ \\
\hline$g$ & 56644.5132 & $8.2198 \pm 0.1750$ & $\mathrm{CrAO}$ \\
\hline$\cdots$ & $\cdots$ & $\cdots$ & ... \\
\hline$r$ & 56682.6001 & $9.9075 \pm 0.0397$ & $\mathrm{LCO} 5$ \\
\hline$r$ & 56683.3380 & $9.8560 \pm 0.0415$ & $\mathrm{LCO} 4$ \\
\hline$r$ & 56684.3056 & $10.1500 \pm 0.0392$ & LCO6 \\
\hline$\ldots$ & $\ldots$ & $\ldots$ & $\ldots$ \\
\hline$i$ & 56639.6672 & $6.9188 \pm 0.0114$ & $\mathrm{CrAO}$ \\
\hline$i$ & 56644.5162 & $7.0678 \pm 0.0096$ & $\mathrm{CrAO}$ \\
\hline$i$ & 56646.5303 & $7.0834 \pm 0.0067$ & $\mathrm{CrAO}$ \\
\hline$\cdots$ & $\cdots$ & $\cdots$ & $\cdots$ \\
\hline$z$ & 56639.6682 & $6.1075 \pm 0.0665$ & $\mathrm{CrAO}$ \\
\hline$z$ & 56643.6256 & $6.4154 \pm 0.0692$ & $\mathrm{CrAO}$ \\
\hline$z$ & 56644.5170 & $6.3643 \pm 0.0238$ & $\mathrm{CrAO}$ \\
\hline$\ldots$ & $\cdots$ & $\cdots$ & $\ldots$ \\
\hline
\end{tabular}

(This table is available in its entirety in machine-readable form.)

the $5100 \AA$ light curve uncertainties are unlikely to be underestimated (see the detailed explanation in Fausnaugh 2017). For the ICCF centroid distributions, the median lag did not change, although the width of the distributions increased. For the JAVELIN models, the posterior lag distributions shifted closer to zero lag-for the $\times 1.5$ rescaling, the lag is $0.36 \pm 0.20$ days $(1.8 \sigma)$, whereas for the $\times 3.0$ rescaling, the lag is $0.22 \pm 0.25$ days $(0.9 \sigma)$.

This seems to indicate that the $g$-band lag is an artifact. However, it is peculiar that the ICCF centroid, which relies on different assumptions than JAVELIN and is less dependent on the measurement uncertainties, should consistently be skewed away from zero. Further investigation showed that the positive $g$-band-5100 $\AA$ lag signal is weakly present in both the LCO and $\mathrm{CrAO}$ light curves independently, with a lag of $0.33 \pm 0.35$ days for $\mathrm{LCO}$ and $0.31 \pm 0.15$ days for CrAO (rest frame). We also tried fitting the $V$-band data (which tends to have smaller uncertainties) separately from the MDM spectroscopic $5100 \AA$ light curve. This still yielded a positive $g$-band lag, with values of $0.71 \pm 0.12$ days $(5.9 \sigma)$ relative to the $V$-band and $0.42 \pm 0.15$ days $(2.8 \sigma)$ relative to the $5100 \AA$ continuum.

Inspection of Table 6 and Figure 1 show that the lagwavelength relation through the ugriz bands is monotonic. Thus, another possibility is that the $5100 \AA$ light curve is an outlier and these results are related to using this light curve as the driver. To test this, we re-ran the FR/RSS procedure and the JAVELIN models using the $g$-band as the driving light curve. However, this made no change except to shift all of the observed lags by precisely the $g$-band $-5100 \AA$ lag.

Thus, there is some evidence that the lag is a real signal in the data. A possible explanation is bias by BLR emission,
Table 4

NGC 2617 Swift Light Curves

\begin{tabular}{|c|c|c|}
\hline Filter & $\begin{array}{c}\mathrm{HJD}-2,400,000 \\
\text { (days) }\end{array}$ & $\begin{array}{c}F_{\lambda} \\
\left(10^{-15} \mathrm{erg} \mathrm{cm}^{-2} \mathrm{~s}^{-1} \AA^{-1}\right)\end{array}$ \\
\hline $\mathrm{X}$-rays ${ }^{\mathrm{a}}$ & 56413.9240 & $2.8400 \pm 0.1200$ \\
\hline $\mathrm{X}$-rays & 56415.2573 & $2.2900 \pm 0.1200$ \\
\hline $\mathrm{X}$-rays & 56415.6885 & $2.3400 \pm 0.1200$ \\
\hline$\cdots$ & $\cdots$ & $\cdots$ \\
\hline$U V W 2$ & 56413.9240 & $12.1448 \pm 0.5593$ \\
\hline$U V W 2$ & 56415.2573 & $12.3706 \pm 0.6836$ \\
\hline$U V W 2$ & 56415.6885 & $11.9232 \pm 0.6589$ \\
\hline$\cdots$ & $\cdots$ & $\ldots$ \\
\hline$U V M 2$ & 56413.9240 & $11.9514 \pm 0.6605$ \\
\hline$U V M 2$ & 56415.2573 & $12.2862 \pm 0.6790$ \\
\hline$U V M 2$ & 56415.6885 & $12.0620 \pm 0.6666$ \\
\hline$\cdots$ & $\cdots$ & $\cdots$ \\
\hline$U V W 1$ & 56413.9240 & $10.6184 \pm 0.5868$ \\
\hline$U V W 1$ & 56415.2573 & $10.9159 \pm 0.6032$ \\
\hline$U V W 1$ & 56415.6885 & $10.5211 \pm 0.5814$ \\
\hline$\cdots$ & $\cdots$ & $\cdots$ \\
\hline$u$ & 56413.9240 & $8.8754 \pm 0.4087$ \\
\hline$u$ & 56415.2573 & $8.6335 \pm 0.3976$ \\
\hline$u$ & 56415.6885 & $8.4759 \pm 0.3903$ \\
\hline$\cdots$ & $\cdots$ & $\cdots$ \\
\hline$b$ & 56413.9240 & $6.9168 \pm 0.3185$ \\
\hline$b$ & 56415.2573 & $6.6667 \pm 0.3070$ \\
\hline$b$ & 56415.6885 & $6.7283 \pm 0.3099$ \\
\hline$\cdots$ & $\cdots$ & $\cdots$ \\
\hline$v$ & 56413.9240 & $6.5059 \pm 0.2397$ \\
\hline$v$ & 56415.2573 & $6.6268 \pm 0.2441$ \\
\hline$v$ & 56415.6885 & $6.5059 \pm 0.2397$ \\
\hline$\ldots$ & $\ldots$ & $\ldots$ \\
\hline
\end{tabular}

Note.

${ }^{\mathrm{a}} 0.3-10 \mathrm{keV}$ absorption-corrected flux $\left(10^{-11} \mathrm{erg} \mathrm{cm}^{-2} \mathrm{~s}^{-1}\right)$.

(This table is available in its entirety in machine-readable form.)

similar to the $u$-band and Swift $u$-band in NGC 2617. The $g$-band is contaminated by both the $\mathrm{H} \beta$ and $H \gamma$ broad lines in MCG+08-11-011, while the spectroscopic data and $V$-band are virtually free of line emission (there may be a small amount of BLR contamination by Fe II emission at these wavelengths). Using the synphot package in IRAF to estimate broadband fluxes from the mean MDM spectrum, we find that the Balmer lines contribute only $7 \%$ of the total $g$-band continuum flux, so it would be surprising if line emission had a large effect on the observed lag. However, Fausnaugh et al. (2016) found that the bias from BLR emission depends more strongly on the variability amplitude of the line emission, which is quite large in this object (the Balmer lines display fractional variability amplitudes $F_{\text {var }}>7 \%-9 \%$, Fausnaugh et al. 2017). Thus, it is not out of the question that the 0.2-0.5 day lag is biased by BLR emission. If a similar bias exists in the other broadband filters, this may explain why the $5100 \AA$ light curve appears as an outlier from the lag-wavelength relation. The $u$ - and $r$-bands are contaminated by Balmer continuum emission and $\mathrm{H} \alpha$, respectively, while the Paschen continuum may be significant in the $i$ - and $z$-bands (Korista \& Goad 2001). 
Table 5

Light-curve Properties

\begin{tabular}{|c|c|c|c|c|c|c|c|}
\hline $\begin{array}{l}\text { Object } \\
\text { (1) }\end{array}$ & $\begin{array}{l}\text { Light } \\
\text { Curve } \\
\text { (2) }\end{array}$ & $\begin{array}{r}N_{\text {obs }} \\
\text { (3) }\end{array}$ & $\begin{array}{c}\Delta t_{\text {med }} \\
\text { (days) } \\
\text { (4) }\end{array}$ & $\begin{array}{c}\hat{F} \\
(5)\end{array}$ & $\begin{array}{c}\langle\mathrm{S} / \mathrm{N}\rangle \\
(6)\end{array}$ & $\begin{array}{l}F_{\text {var }} \\
(7)\end{array}$ & $\begin{array}{c}(\mathrm{S} / \mathrm{N})_{\mathrm{var}} \\
(8)\end{array}$ \\
\hline \multirow{5}{*}{$\begin{array}{c}\mathrm{MCG}+08- \\
11-011\end{array}$} & $u$ & 41 & 1.54 & 6.30 & 44.3 & 0.10 & 19.0 \\
\hline & $g$ & 85 & 0.99 & 7.47 & 202.2 & 0.07 & 83.3 \\
\hline & $r$ & 42 & 1.07 & 9.17 & 194.4 & 0.05 & 41.0 \\
\hline & $i$ & 41 & 1.07 & 7.01 & 231.7 & 0.04 & 41.1 \\
\hline & $z$ & 41 & 1.07 & 6.23 & 225.5 & 0.03 & 33.5 \\
\hline \multirow[t]{12}{*}{ NGC 2617} & X-rays & 136 & 1.20 & 4.00 & 20.8 & 0.57 & 91.2 \\
\hline & $U V W 2$ & 126 & 1.12 & 13.50 & 18.9 & 0.40 & 59.2 \\
\hline & UVM2 & 126 & 1.27 & 12.50 & 17.6 & 0.36 & 50.3 \\
\hline & $U V W 1$ & 131 & 1.09 & 11.00 & 18.8 & 0.29 & 42.9 \\
\hline & Swift $u$ & 130 & 1.10 & 8.89 & 21.5 & 0.29 & 49.2 \\
\hline & Swift $b$ & 129 & 1.11 & 6.94 & 21.6 & 0.16 & 26.9 \\
\hline & Swift $v$ & 119 & 1.14 & 6.43 & 22.2 & 0.09 & 15.3 \\
\hline & $u$ & 113 & 0.66 & 7.11 & 77.5 & 0.10 & 56.6 \\
\hline & $g$ & 166 & 0.56 & 8.38 & 275.1 & 0.04 & 83.8 \\
\hline & $r$ & 127 & 0.62 & 10.60 & 319.2 & 0.04 & 82.4 \\
\hline & $i$ & 154 & 0.60 & 8.28 & 287.7 & 0.02 & 46.9 \\
\hline & $z$ & 153 & 0.59 & 8.42 & 230.7 & 0.02 & 41.6 \\
\hline
\end{tabular}

Note. Column 3 gives the number of observations in each light curve. Column 4 gives the median cadence. Column 5 gives the mean flux level of each light curve in units of $10^{-15} \mathrm{erg} \mathrm{cm}^{-2} \mathrm{~s}^{-1} \AA^{-1}$ (the X-rays are units of $10^{-11} \mathrm{erg} \mathrm{cm}^{-2} \mathrm{~s}^{-1}$ ). Column 6 gives the mean signal-to-noise ratio $\langle\mathrm{S} / \mathrm{N}\rangle$. Column 7 gives the rms fractional variability, defined in Section 2.2. Column 8 gives the approximate $\mathrm{S} / \mathrm{N}$ at which we detect variability (Section 2.2).

\section{CREAM Modeling}

We also analyzed the light curves using the Continuum REprocessed AGN Markov Chain Monte Carlo code (CREAM, Starkey et al. 2016). CREAM fits an accretion disk reprocessing model directly to the observed light curves, so as to estimate the temperature profile of the disk and its inclination to the observer's line of sight. The adopted geometry is a "lamp post," which attributes the primary emission to a point source at a small distance above the central black hole. As the lamp post varies, it irradiates the disk, which thermally reprocesses the incident flux into variations at longer wavelengths. Physically, the point source may correspond to the X-ray emitting corona, if the corona is small compared to the size of the disk. However, a physical interpretation of the lamp post is not required-this geometry should reasonably approximate most models that place the origin of driving emission near the $\mathrm{SMBH}$ at a small height above the disk midplane. This includes models like those of Luo \& Liang (1998) and Nealon et al. (2015), which modify the structure of the disk on scales of $\sim 10 R_{\mathrm{g}}$.

CREAM fits the model by inferring the transfer functions and driving lamp-post light curve that best reproduces the observed data. The transfer functions are calculated from a thin accretion disk with three parameters: the temperature $T_{0}$ at the inneredge, the index $\beta$ of a power-law temperature profile, and the inclination $i$ of the disk to the observer's line of sight. CREAM takes a Bayesian approach, sampling the posterior probability distributions of the driving light curve and disk parameters.
With an estimate of $T_{0}$, it is then possible to calculate the product $M_{\mathrm{BH}} \dot{M}$, where $M_{\mathrm{BH}}$ is the mass of the black hole and $\dot{M}$ is the mass accretion rate through the disk (see Cackett et al. 2007 and Starkey et al. 2016 for a derivation).

For our first model, we fixed the power-law index $\beta$ to $-3 / 4$ and held the inclination $i$ of the disk constant at $0^{\circ}$. In Figure 4, we show the posterior distributions of $M_{\mathrm{BH}} \dot{M}$ for the two AGNs (we show fits to the transfer functions of individual light curves and the inferred driving light curves in the Appendix). CREAM finds $\log M_{\mathrm{BH}} \dot{M}=6.63 \pm 0.24\left[M_{\odot}^{2} \mathrm{yr}^{-1}\right]$ in MCG+08-11-011, and $\log M_{\mathrm{BH}} \dot{M}=5.58 \pm 0.21\left[M_{\odot}^{2} \mathrm{yr}^{-1}\right]$ in NGC 2617. We then ran models that allowed the inclination to vary (owing to the short wavelength baseline spanned by our light curves, we were unable to place meaningful constraints on $\beta$ ). For $\mathrm{MCG}+08-11-011$, we were unable to constrain the inclination, but we found $i=43^{\circ} \pm 20^{\circ}$ with $\log M_{\mathrm{BH}} \dot{M}=5.24 \pm 0.23\left[M_{\odot}^{2} \mathrm{yr}^{-1}\right]$ for NGC 2617 .

We compare the CREAM results to our time-series analysis from Section 3 by giving the mean lags of the transfer functions in Tables 6 and 7. Because we use the $5100 \AA$ light curve as a reference in Section 3, we subtract this lag from the other CREAM values in these tables. For NGC 2617, there is excellent agreement between the CREAM results and the lags estimated from $\tau_{\text {cent }}$ and $\tau_{\text {JAV }}$. For MCG+08-11-011, the CREAM lags are shifted by about $0.5-1.0$ days relative to the values of $\tau_{\text {JAV }}$. As noted in Section 3.1, the $g$-band-5100 $\AA$ lag from our time series analysis may be an outlier, while the temperature gradient in CREAM's physical model forces $\tau \propto \lambda^{4 / 3}$ and an anomalous $g$-band-5100 $\AA$ lag cannot be produced. Using the $g$-band as the reference wavelength (subtracting the $g$-band lag from the other lags in Columns 3-6 of Table 6), we find much better agreement. This calculation is explicitly shown for $\tau_{\text {JAV }}$ in Table 6 . The reduced $\chi^{2}$ values of the CREAM fits are larger than would be expected for Gaussian statistics (1.96-2.37), which may indicate that the light curve uncertainties are underestimated, or that the model is not a perfect description of the data.

Fausnaugh et al. (2017) estimated black hole masses for these objects, which allows us to calculate $\dot{M}$ from the CREAM fits (Table 1). For MCG+08-11-011, $M_{\mathrm{BH}} \sim 2.82 \times 10^{7} M_{\odot}$, implying $\dot{M}=0.151 M_{\odot} \mathrm{yr}^{-1}$ and an Eddington ratio $\dot{m}_{\mathrm{Edd}}=$ $\dot{M} / \dot{M}_{\mathrm{Edd}}=0.234$ with $\eta=0.1$. For NGC $2617, M_{\mathrm{BH}} \sim$ $3.24 \times 10^{7} M_{\odot}$, and we calculate $\dot{M}=0.012 M_{\odot} \mathrm{yr}^{-1}$ and $\dot{m}_{\text {Edd }}=0.016$.

These Eddington ratios can be compared to independent estimates using the observed luminosities during the monitoring campaign (Table 1 , again, we assume that $\dot{M} / \dot{M}_{\text {Edd }}=$ $\left.L / L_{\text {Edd }}, L=10 \lambda L_{5100} \AA\right) .{ }^{50}$ The estimates of the Eddington ratios from the CREAM models are a factor of 4.3 larger for MCG+08-11-011 and a factor 1.6 larger for NGC 2617. However, there are large uncertainties associated with these estimates. Runnoe et al. (2012) empirically find a bolometric correction of $8.1 \pm 0.4$, but recommend estimating the bolometric luminosity with the relation $L \approx 10^{4.9}$ $\left(\lambda L_{5100 \AA}\right)^{0.9}$, with an intrinsic scatter of 0.17 dex around this

\footnotetext{
50 With the multi-wavelength coverage for NGC 2617, we also estimated the bolometric correction by integrating the observed mean fluxes corrected for Galactic extinction and then dividing by the mean of $L_{5100}$. This yields a bolometric correction of 9.3. Alternatively, we fit the composite QSO template of Vanden Berk et al. (2001) to the UV data, and integrated the X-rays and template through $1 \mu \mathrm{m}$. This yields a slightly smaller bolometric correction of 8.9 , because this method is not affected by host-galaxy light.
} 
Table 6

MCG+08-11-011 Rest-frame Continuum Lags

\begin{tabular}{|c|c|c|c|c|c|c|}
\hline $\begin{array}{l}\text { Filter } \\
\text { (1) }\end{array}$ & $\begin{array}{l}\lambda \\
(\AA) \\
(2)\end{array}$ & $\begin{array}{r}\tau_{\text {cent }} \\
\text { (days) } \\
\text { (3) }\end{array}$ & $\begin{array}{r}\tau_{\text {peak }} \\
\text { (days) } \\
(4)\end{array}$ & $\begin{array}{r}\tau_{\text {JAV }} \\
\text { (days) } \\
(5)\end{array}$ & $\begin{array}{c}\tau_{\text {CREAM }^{-}-\tau_{5100 \AA}} \\
\text { (days) } \\
\text { (6) }\end{array}$ & $\tau_{\text {JAV }}-\tau_{\varepsilon}$ \\
\hline $\bar{u}$ & 3449 & $0.66_{-0.60}^{+0.68}$ & $-0.05_{-1.30}^{+0.86}$ & $-0.04_{-0.32}^{+0.30}$ & $-0.52 \pm 0.16$ & $-0.54_{-0.32}^{+0.30}$ \\
\hline $5100 \AA$ & 5100 & $-0.01_{-0.31}^{+0.30}$ & $0.00_{-0.14}^{+0.14}$ & $\ldots$ & $0.00 \pm 0.27$ & -0.50 \\
\hline$r$ & 6124 & $2.49_{-0.51}^{+0.49}$ & $2.16_{-0.48}^{+0.72}$ & $1.19_{-0.15}^{+0.16}$ & $0.24 \pm 0.33$ & $0.69_{-0.15}^{+0.16}$ \\
\hline
\end{tabular}

Note. Column 2 gives the rest-frame effective wavelength of the filter. Column 3 gives the ICCF centroids and the $68 \%$ confidence intervals from the FR/RSS procedure (see Section 3). Column 4 gives the same, but for the ICCF peaks. Column 5 gives the lags fit by JAVELIN and the central $68 \%$ confidence interval of the posterior distributions. Column 6 gives the lag estimates from the transfer functions fit by CREAM (see Section 4). Column 7 is the same as Column 5, but with the $g$ band lag subtracted. All lag values have been corrected to the rest-frame.

relation. The first option would decrease the value of $\dot{m}_{\text {Edd }}$ (as estimated from the optical luminosity) by about $20 \%$, while the second estimate would increase $\dot{m}_{\text {Edd }}$ by $20 \%$ and $38 \%$ in MCG+08-11-011 and NGC 2617, respectively. Thus, there is at least a factor of 1.2-1.3 systematic uncertainty on $\dot{m}_{\text {Edd }}$ as estimated from the optical luminosity, with an additional factor of 1.5 statistical uncertainty due to the intrinsic scatter. The widths of the $68 \%$ confidence intervals of the posterior distributions of $M \dot{M}$ also imply a factor of $\sim 1.7$ uncertainty on the estimate of $\dot{m}_{\text {Edd }}$ from CREAM. Furthermore, the estimate of $\dot{m}_{\text {Edd }}$ from CREAM depends on the adopted SMBH mass, which is intrinsically uncertain by a factor of 2.5 to 3.0. Finally, the estimate of $\dot{m}_{\text {Edd }}$ from CREAM depends on the choice of $\eta$. Although $\eta$ can vary between about 0.06 and 0.50 , depending on the spin of the black hole, it is most likely that the black hole is co-rotating with the disk. This suggests that $\eta \geqslant 0.1$, which decreases $\dot{M}_{\text {Edd }}$ and increases the discrepancy in $\dot{m}_{\text {Edd }}$. We therefore ignore uncertainty in $\eta$ when calculating the significance of the discrepancies, noting that uncertainty in this parameter will tend to strengthen our results.

Combining the uncertainties on the bolometric correction in quadrature, $M \dot{M}$ from $\mathrm{CREAM}$, and $M_{\mathrm{BH}}$, there is a total uncertainty of about $0.56 \mathrm{dex}$. The factor of 4.3 disagreement in MCG+08-11-011 can then be written as $0.64 \pm 0.56 \mathrm{dex}$, while the factor of 1.6 disagreement in NGC 2617 is $0.19 \pm 0.56$ dex. The estimate of $\dot{m}_{\text {Edd }}$ from CREAM for NGC 2617 therefore appears to be consistent with the observed optical luminosity, and there is only a small difference for MCG+08-11-011.

\section{Discussion}

\subsection{Comparison with Previous Studies}

MCG+08-11-011 is one of the two objects with statistically significant continuum lags based on data from $\mathrm{CrAO}$ taken between 2001 and 2003, with an approximately three-day cadence excepting seasonal gaps (Sergeev et al. 2005). Based on the centroid of the cross-correlation functions (using the same ICCF and FR/RSS methods employed in this study), Sergeev et al. (2005) found lags for the $V-, R$-, and $I$-bands (relative to the $B$-band) of $0.91 \pm 0.53$ days, $4.64 \pm 0.81$, and $5.75 \pm 1.18$ days (rest-frame), respectively. This result accords with the expected trend of larger lags at longer wavelengths. However, the magnitude of the lags is several days larger than those measured here, which range between about 1 and 1.5 days from the $g$-band to the $i$ - and $z$ bands. While it is possible that the structure of the disk has changed over the past decade, it is more likely that the lower cadence and large gaps in the Sergeev et al. (2005) light curves result in larger uncertainties than estimated in that study. The light curves presented here have no seasonal gaps and daily cadence, which should yield more reliable lags.

Interband continuum lags were detected in NGC 2617 by Shappee et al. (2014). After the All Sky Automated Survey for Supernovae (ASAS-SN ${ }^{51}$ ) observed a sudden X-ray/optical outburst of this AGN in 2013, intensive multi-wavelength monitoring of the target ensued for $\sim 50$ days. This target-ofopportunity campaign was led by X-ray and near-UV observations from the Swift satellite, while ground-based monitoring extended the wavelength coverage through optical and near-IR wavelengths. The observed lags, relative to the $V$-band, ranged from $-1.11 \pm 0.32$ days in the $U V W 2$ filter to $1.97 \pm 1.32$ days in the $I$-band and $7.42 \pm 1.25$ days in the $K$-band (rest-frame). There was also a $3.36 \pm 0.42 \mathrm{X}$-ray lag (or a $\sim 2.2$ day lag between the X-rays and $U V W 2$ ). These lags were measured with JAVELIN in the same way as in this study, but using the Swift X-ray and UVW2 light curves as drivers.

The $U V W 2-5100 \AA$ lag measured here is almost a factor of 3 smaller than that measured by Shappee et al. (2014), although this is only a $2.3 \sigma$ difference. We find that the $i$ - and $z$-band lags from the 2014 data are also smaller than in 2013 by a factor of about $2(1.0 \sigma)$. The X-ray lag is 0.78 days $(1.9 \sigma)$ shorter than that reported by Shappee et al. (2014).

Thus, the lags measured here are broadly consistent with those reported by Shappee et al. (2014), but systematically smaller. Under the standard RM formalism, the observed lag between two light curves is not independent of the driving light curve autocorrelation function (Blandford \& McKee 1982; Peterson 1993). The variability amplitude of NGC 2617 in 2013 was much stronger than in 2014 , and the timescale of variations is smaller in 2014 than in 2013. Both of these factors will generally lead to smaller lags, which may account for these results. This is similar to the results of Goad \& Korista (2014), who find that lags in the BLR will be observed to be smaller for weak and rapid variations simply due to geometric dilution. Another possibility is that the physical configuration of the disk has changed-because NGC 2617 is a "changing look" AGN,

\footnotetext{
51 http://www.astronomy.ohio-state.edu/ assassin
} 
Table 7

NGC 2617 Rest-frame Continuum Lags

\begin{tabular}{|c|c|c|c|c|c|}
\hline $\begin{array}{l}\text { Filter } \\
\text { (1) }\end{array}$ & $\begin{array}{c}\lambda \\
(\AA) \\
(2)\end{array}$ & $\begin{array}{r}\tau_{\text {cent }} \\
\text { (days) } \\
(3)\end{array}$ & $\begin{array}{r}\tau_{\text {peak }} \\
\text { (days) } \\
(4)\end{array}$ & $\begin{array}{r}\tau_{\text {JAV }} \\
\text { (days) } \\
(5)\end{array}$ & $\begin{array}{c}\tau_{\text {CREAM }}-\tau_{5100 \AA} \\
\text { (days) } \\
(6)\end{array}$ \\
\hline X-rays & 9 & $-2.47_{-0.81}^{+0.88}$ & $-2.48_{-1.17}^{+1.22}$ & $-2.58_{-0.08}^{+0.09}$ & $\ldots$ \\
\hline$U V M 2$ & 2214 & $-0.66_{-0.55}^{+0.52}$ & $-0.92_{-0.97}^{+0.49}$ & $-0.25_{-0.28}^{+0.28}$ & $-0.35 \pm 0.03$ \\
\hline$U V W 1$ & 2563 & $-0.29_{-0.64}^{+0.62}$ & $-0.24_{-0.68}^{+0.83}$ & $0.24_{-0.25}^{+0.27}$ & $-0.33 \pm 0.04$ \\
\hline Swift $b$ & 4268 & $-0.12_{-0.74}^{+0.00}$ & $-0.24_{-1.02}^{+0.87}$ & $0.23_{-0.40}^{+0.47}$ & $-0.01 \pm 0.08$ \\
\hline$g$ & 4732 & $0.03_{-0.58}^{+0.58}$ & $0.10_{-0.63}^{+0.92}$ & $-0.16_{-0.18}^{+0.18}$ & $-0.08 \pm 0.09$ \\
\hline $5100 \AA$ & 5100 & $0.02_{-0.47}^{+0.47}$ & $0.00_{-0.19}^{+0.19}$ & $\ldots$ & $0.00 \pm 0.11$ \\
\hline Swift v & 5326 & $-0.46_{-1.02}^{+0.96}$ & $-0.29_{-1.12}^{+1.22}$ & $-0.38_{-0.52}^{+0.64}$ & $0.01 \pm 0.11$ \\
\hline$r$ & 6162 & $0.98_{-1.01}^{+0.91}$ & $0.92_{-0.87}^{+1.51}$ & $0.37_{-0.18}^{+0.17}$ & $0.11 \pm 0.13$ \\
\hline$i$ & 7582 & $0.68_{-0.58}^{+0.82}$ & $0.63_{-0.78}^{+0.92}$ & $0.60_{-0.18}^{+0.20}$ & $0.32 \pm 0.17$ \\
\hline
\end{tabular}

Note. Columns 2 through 6 are the same as in Table 6 . All values have been corrected to the rest-frame.

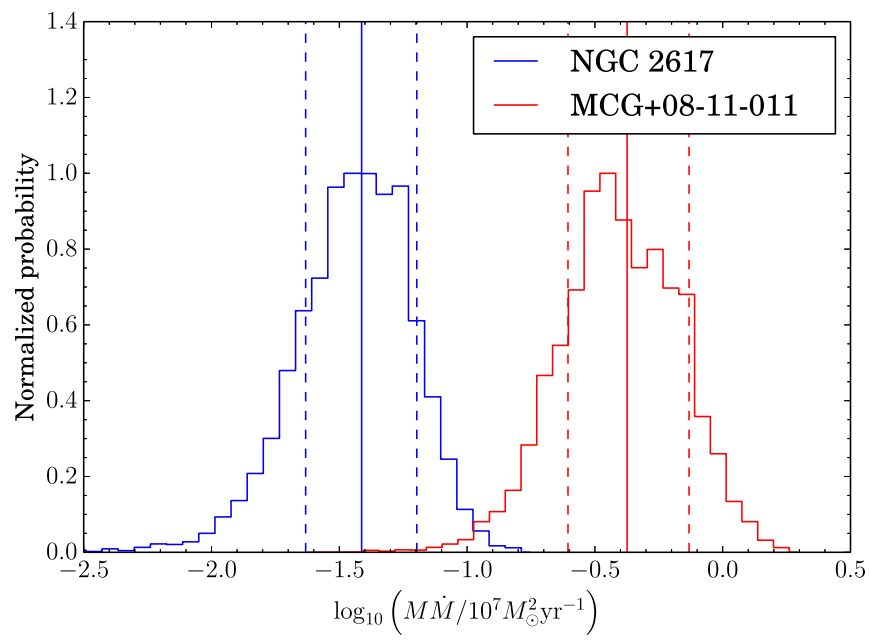

Figure 4. Posterior distributions for $M_{\mathrm{BH}} \dot{M}$ for $\mathrm{MCG}+08-11-011$ (red) and NGC 2617 (blue), as derived by CREAM. The solid lines give the medians of the distributions, and the dashed lines give the $68 \%$ confidence intervals. For these fits, the temperature profile was fixed to $R^{-3 / 4}$ and the inclination to $i=0$.

the accretion flow may be far from equilibrium (LaMassa et al. 2015; MacLeod et al. 2016; Runnoe et al. 2016). The two monitoring programs are separated by one year, and the dynamical time at a distance of one light day from the black hole is about one month. A bulk readjustment of the accretion flow is therefore possible in the time between the two campaigns. The luminosity was also a factor of 1.8 smaller in 2014 compared to 2013, and the size of the disk is expected to scale with luminosity (see Section 5.3 below). However, this adjustment should happen on a viscous timescale, which is on the order of decades to centuries for a typical Seyfert 1 (e.g., LaMassa et al. 2015).

\subsection{Challenges to the Disk Reprocessing Model}

The disk reprocessing model posits that short-wavelength radiation drives long-wavelength emission by heating the accretion disk and perturbing the local temperature. Two important predictions of this model are that the X-ray, UV, and optical light curves will be well-correlated, and that longer wavelength light curves should lag behind shorter wavelength light curves. We qualitatively find results consistent with disk reprocessing - the UV and optical light curves in both objects are well correlated, and the lag-wavelength relation is nearly monotonic.

However, it is clear from visual inspection of the NGC 2617 light curves that there is much more structure in the X-rays than in the UV and optical emission, especially on short timescales. Although the X-ray light curve would be expected to be smoothed if reprocessed at UV wavelengths, comparison of Figures 3 and the Appendix shows that the inferred driving light curve does not correlate very well with the observed X-ray variations (this is confirmed by the ICCF analysis from Section 3). This poor correlation was also seen in 2013 by Shappee et al. (2014), and has been observed in other objects, including NGC 5548 (Uttley et al. 2003; Edelson et al. 2015), MR 2251-178 (Arévalo et al. 2008), Mrk 79 (Breedt et al. 2009), NGC 3783 (Arévalo et al. 2009), and NGC 4151 (Edelson et al. 2017). Several of these studies have been unable to represent the UV/optical light curves as a reprocessed (smoothed and shifted) version of the X-ray light curve (Arévalo et al. 2008; Breedt et al. 2009; Starkey et al. 2017), which is problematic for a generic disk reprocessing model. A notable exception is Shappee et al. (2014), who were able to produce a good, but not perfect, match between the X-ray and optical light curves from 2013 using a simple reprocessing model for NGC 2617. However, they found X-ray to UV/optical lags (2-3 days) that are much larger than any plausible lighttravel time across the accretion disk. Shappee et al. (2014) were unable to provide a physical interpretation for the X-ray-optical lag, and we find a similar X-ray-optical lag here ( $\sim 2.6$ days), reaffirming this problem for the disk reprocessing model.

Although the X-ray light curve in NGC 2617 is problematic for disk reprocessing, this paradigm may still be importantthe UV/optical light curves display strong correlations and follow the prediction of larger lags at longer wavelengths. A possible explanation is that the driving light curve is in the extreme UV (Shakura \& Sunyaev 1973; Gardner \& Done 2017). On the other hand, although the X-ray light curve has additional structure compared to the UV/optical light 
curves, there is still clearly some connection. Considering the temporal lead of the high-energy emission, this suggests a very complicated relationship between the X-rays and UV/optical emission. We stress that there are many possibilities for the geometry and energetics of the X-ray-emitting corona based on both analytic results and simulations, and it is not clear what lag-wavelength relations or variable X-ray emission these models would produce (see, e.g., Schnittman et al. 2013; Jiang et al. 2014 for coronal emission extended across the disk, and Begelman et al. 2015; Sa̧dowski 2016; Begelman \& Silk 2017 for the possible effects of toroidal magnetic fields). However, observational evidence strongly favors a compact corona (Mosquera et al. 2013; Reis \& Miller 2013), which makes some aspects of the reprocessing model very likely, and continuing multi-wavelength monitoring of this and other Seyfert $1 \mathrm{~s}$ is therefore an important avenue for further investigations. For example, see Giustini et al. (2017) for an analysis of the X-ray emission in NGC 2617 during 2013, and Oknyansky et al. (2017) for an analysis of the X-ray through IR emission in 2016.

\subsection{Disk Radii and Temperature Profiles}

Figure 5 shows the lags as a function of wavelength for each object. For a disk reprocessing model, the lag-wavelength relation contains information about the absolute size of the disk and the temperature profile. To quantify this, we fit a model of the form

$$
\tau=\tau_{0}\left[\left(\frac{\lambda}{\lambda_{0}}\right)^{\beta}-1\right],
$$

where $\lambda$ is the effective wavelength transformed to the restframe, $\lambda_{0}$ is some reference wavelength, and $\tau_{0}$ and $\beta$ are free parameters. The normalization $\tau_{0}$ measures the radius of the disk emitting at a reference wavelength $\lambda_{0}$, and the index $\beta$ measures the temperature profile of the disk, $T \propto R^{-1 / \beta}$. Standard thin-disk theory predicts that $\beta=4 / 3$, and assuming that the lags trace the flux-weighted mean radius for emission at $\lambda, \tau_{0}$ scales as

$$
\left(\frac{\tau_{0}}{1.0 \text { days }}\right)=\left(\frac{\lambda_{0}}{4800 \AA}\right)^{4 / 3}\left(\frac{M_{\mathrm{BH}}}{10^{8} M_{\odot}}\right)^{2 / 3}\left(\frac{\dot{m}_{\mathrm{Edd}}}{0.09}\right)^{1 / 3}
$$

(Fausnaugh et al. 2016). This calculation assumes both a radiative efficiency $\eta=L_{\mathrm{Bol}} / \dot{M} c^{2}=0.10$ and that the X-ray/ far-UV radiation does not appreciably heat the disk compared to viscous dissipation. There can be deviations from this thindisk model on small scales ( $\sim 10 R_{\mathrm{g}}$ or a few light hours, e.g., Luo \& Liang 1998; Noble et al. 2011; Schnittman et al. 2016), which would only result in a small modification to the lagwavelength relation on the scales observed here (several light days).

We fit models with both $\tau_{0}$ and $\beta$ free to vary, as well as with $\beta$ fixed to $4 / 3$. We set the reference wavelength to $\lambda_{0}=4800 /(1+z) \AA$, the rest-frame $g$-band effective wavelength. To match the model so that the $g$-band lag equals 0 , we subtracted the $g$-band-5100 $\AA$ lag from all measurements of $\tau_{\text {JAV }}$ (as discussed in Section 3.1, this is equivalent to fitting the lags using the $g$-band as the driver). We also tested fits where we excluded suspect lag measurements. In particular, we tried omitting the $u$-band lag for both targets because this lag is probably contaminated by Balmer continuum emission from the BLR. For MCG+08-11-011, we also tried omitting the anomalous $g$-band-5100 $\mathrm{\AA}$ lag, which is likely an outlier. For NGC 2617, we tested models that jointly fit the Swift and ground-based data, as well as separate fits to each respective data set. We also excluded the large X-ray lag for this object, because the lag is much larger than any plausible lighttravel time.

The results of these fits are given in Table 8 . Because of the large uncertainties and limited amount of data, the fits sometimes prefer a flat relation $(\beta=0)$ that does not provide any constraint on $\tau_{0}$. For the fits that do converge, the uncertainties on $\tau_{0}$ and $\beta$ are still very large and do not put interesting physical constraints on the disk. We include the results of these fits for completeness, but the rest of our discussion focuses on the fits for $\tau_{0}$ assuming $\beta=4 / 3$.

Fits to the ICCF and JAVELIN lags give consistent values of $\tau_{0}$, although the ICCF fits are poorly constrained. Excluding the anomalous $g$-band-5100 $\AA$ lag in MCG+08-11-011 results in reasonable values of $\chi^{2} /$ dof (where dof is the number of degrees of freedom in the fit) between 0.75 and 1.01 , with dof between 3 and 4 . For NGC 2617, including the $u$-band and Swift $u$-band lags gives $\chi^{2} /$ dof of 1.67-2.66 with dof between 5 and 11, somewhat larger than would be expected for Gaussian statistics. Censoring these lags results in a $\chi^{2} /$ dof between 0.79 and 1.16. Excluding all of the Swift data increases $\tau_{0}$ from 0.38 days to 0.51 days, while excluding the groundbased data decreases $\tau_{0}$ to below 0.19 light days. This can be understood by considering the very small UV lags that are only detected at $\sim 1 \sigma$. Including these data moves the model to smaller $\tau_{0}$, consistent with the unresolved lags, while the wellresolved ground-based lags pull $\tau_{0}$ to larger values.

The fits with $\chi^{2} /$ dof $\sim 1$ indicate that a disk reprocessing model with $\beta=4 / 3$ can reproduce our data very well. This is consistent with the prediction for a geometrically thin disk with a temperature profile $T \propto R^{-3 / 4}$. This signature power law is difficult to reproduce if the disk is not geometrically thin, and it is not immediately clear what other configurations could mimic the $\tau \propto \lambda^{4 / 3}$ relation. For $\mathrm{MCG}+08-11-011$, an acceptable $\chi^{2} /$ dof requires the removal of the $g$-band-5100 $\AA$ lag, while we must exclude both the ground-based and Swift $u$-bands for NGC 2617. As discussed above, we already suspect that these lags are unreliable, so we adopt final measurements for the disk sizes of $\tau_{0}=1.15 \pm 0.11$ days in $\mathrm{MCG}+08-11-011$ and $\tau_{0}=0.50 \pm 0.12$ days in NGC 2617. These uncertainties represent only the formal errors in the fit.

Using the black hole masses and accretion rates in Table 1, we can predict $\tau_{0}$ using Equation (7). These values are given in Table 8 alongside our fits, and the predicted lag-wavelength relations are shown by the dotted-dashed blue lines in Figure 5. We find that our fits for $\tau_{0}$ are much larger than these predictions. For MCG+08-11-011, the disk is a factor of 3.3 larger (a 7.2 $\sigma$ result), while for NGC 2617 the disk is a factor of 2.3 larger (a $2.3 \sigma$ result).

It is unclear if uncertainties in $M_{\mathrm{BH}}$ and $\dot{m}_{\mathrm{Edd}}$ can explain these discrepancies. A factor of 3.3 increase in $\tau_{0}$ for MCG $+08-11-011$ requires a factor of 36 increase in the product $M_{\mathrm{BH}}^{2} \dot{m}_{\mathrm{Edd}}$, and a factor of 12 increase for a factor of 2.3 in $\tau_{0}$ in the case of NGC 2617. Even if the values of $M_{\mathrm{BH}}$ from Fausnaugh et al. (2017) are underestimated by a factor of 3 (approximately equivalent to the intrinsic scatter in the mean virial factor $\langle f\rangle$, Onken et al. 2004), the optical luminosity 

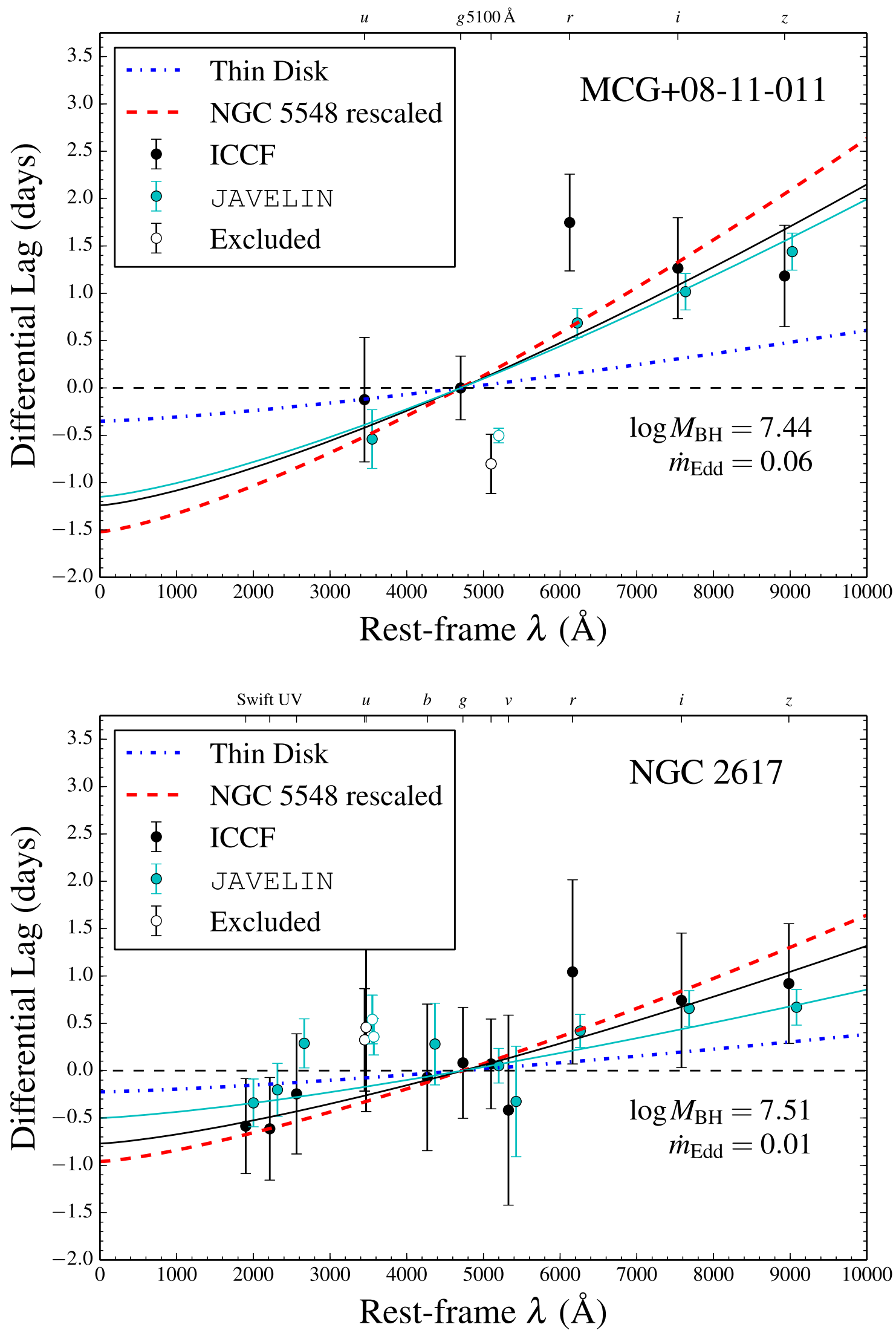

Figure 5. Lag-wavelength relations for each object relative to the $g$-band. The lags from the ICCF are shown in black and those from JAVELIN are shown in cyan. The best fits of $\tau_{0}$ with $\beta=4 / 3$ are shown by the black and cyan lines (see Section 5.3). The predictions from standard thin-disk theory (Equation (7)) are shown by the dotted-dashed blue lines, while the accretion disk in NGC 5548 (Fausnaugh et al. 2016), rescaled to the mass and mass accretion rate of these objects, is shown by the dashed red lines. 
Table 8

Disk Parameter Fits

\begin{tabular}{|c|c|c|c|c|c|c|c|c|c|c|}
\hline Object & $\begin{array}{c}\alpha_{\mathrm{CCF}} \\
\text { (light days) } \\
(2)\end{array}$ & $\beta_{\mathrm{CCF}}$ & $\begin{array}{c}\chi_{\mathrm{CCF}}^{2} / \mathrm{dof} \\
\text { (4) }\end{array}$ & $\begin{array}{l}\rho_{\mathrm{CCF}} \\
\\
(5)\end{array}$ & $\begin{array}{c}\alpha_{\text {JAV }} \\
\text { (light days) } \\
(6)\end{array}$ & $\begin{array}{c}\beta_{\text {JAV }} \\
\text { (7) }\end{array}$ & $\begin{array}{c}\chi_{\text {JAV }}^{2} / \text { dof } \\
\text { (8) }\end{array}$ & $\begin{array}{c}\rho_{\text {JAV }} \\
\\
(9)\end{array}$ & $\begin{array}{c}\text { Thin Disk } \\
\text { (light } \\
\text { days) } \\
(10)\end{array}$ & $\begin{array}{c}\text { NGC } 5548 \\
\text { Rescaled } \\
\text { (light days) } \\
\quad(11)\end{array}$ \\
\hline $\begin{array}{c}\mathrm{MCG}+08- \\
11-011\end{array}$ & $1.13 \pm 0.31$ & $4 / 3$ & 3.16 & $\ldots$ & $1.00 \pm 0.11$ & $4 / 3$ & 16.8 & $\ldots$ & 0.35 & 1.52 \\
\hline no $5100 \AA$ & $1.24 \pm 0.32$ & $4 / 3$ & 1.72 & $\ldots$ & $1.15 \pm 0.11$ & $4 / 3$ & 0.75 & $\ldots$ & & \\
\hline no $u$ & $1.15 \pm 0.32$ & $4 / 3$ & 3.91 & $\ldots$ & $0.99 \pm 0.11$ & $4 / 3$ & 22.26 & $\ldots$ & & \\
\hline no $u / 5100 \AA$ & $1.26 \pm 0.32$ & $4 / 3$ & 2.23 & $\ldots$ & $1.15 \pm 0.11$ & $4 / 3$ & 1.01 & $\ldots$ & & \\
\hline NGC 2617 & $0.65 \pm 0.32$ & $4 / 3$ & 0.27 & $\ldots$ & $0.38 \pm 0.11$ & $4 / 3$ & 2.31 & $\ldots$ & 0.22 & 0.96 \\
\hline no Swift & $0.71 \pm 0.47$ & $4 / 3$ & 0.27 & $\ldots$ & $0.51 \pm 0.13$ & $4 / 3$ & 2.66 & $\ldots$ & & \\
\hline no $u /$ Swift & $0.79 \pm 0.48$ & $4 / 3$ & 0.17 & $\ldots$ & $0.61 \pm 0.14$ & $4 / 3$ & 0.79 & $\ldots$ & & \\
\hline no ugriz & $0.60 \pm 0.44$ & $4 / 3$ & 0.26 & $\ldots$ & $0.05 \pm 0.21$ & $4 / 3$ & 1.67 & $\ldots$ & & \\
\hline no ugriz/Swift $u$ & $0.75 \pm 0.46$ & $4 / 3$ & 0.08 & $\ldots$ & $0.19 \pm 0.22$ & $4 / 3$ & 0.97 & $\ldots$ & & \\
\hline no $u /$ Swift $u$ & $0.77 \pm 0.34$ & $4 / 3$ & 0.12 & $\ldots$ & $0.50 \pm 0.12$ & $4 / 3$ & 1.16 & $\ldots$ & & \\
\hline $\begin{array}{c}\mathrm{MCG}+08- \\
11-011\end{array}$ & $1.86 \pm 6.34$ & $0.92 \pm 2.47$ & 3.94 & -1.0 & $0.20 \pm 0.18$ & $3.38 \pm 1.33$ & 21.07 & -1.0 & & \\
\hline no $5100 \AA$ & $\infty$ & $0.00 \pm 99.9$ & 2.05 & 0.0 & $\infty$ & $0.14 \pm 0.90$ & 0.32 & -1.0 & & \\
\hline no $u$ & $\infty$ & $0.00 \pm 3.81$ & 5.10 & -1.0 & $0.16 \pm 0.16$ & $3.70 \pm 1.44$ & 30.69 & -1.0 & & \\
\hline no $u / 5100 \AA$ & $\infty$ & $0.00 \pm 99.9$ & 2.56 & -1.0 & $\infty$ & $0.00 \pm 99.9$ & 0.40 & 0.0 & & \\
\hline NGC 2617 & $0.62 \pm 0.64$ & $1.55 \pm 1.44$ & 0.29 & -0.9 & $0.09 \pm 0.12$ & $3.84 \pm 2.07$ & 2.01 & -1.0 & & \\
\hline no Swift & $0.23 \pm 1.25$ & $2.90 \pm 8.02$ & 0.31 & -1.0 & $0.15 \pm 0.26$ & $3.12 \pm 2.60$ & 2.99 & -1.0 & & \\
\hline no $u /$ Swift & $\infty$ & $0.00 \pm 99.9$ & 0.19 & 0.0 & $\infty$ & $0.00 \pm 99.9$ & 0.61 & 0.0 & & \\
\hline no ugriz & $\infty$ & $0.00 \pm 99.9$ & 0.25 & 0.0 & $\infty$ & $0.00 \pm 7.16$ & 2.02 & -1.0 & & \\
\hline no ugriz/Swift $u$ & $\infty$ & $0.00 \pm 99.9$ & 0.09 & 0.0 & $\infty$ & $0.00 \pm 99.9$ & 1.20 & -1.0 & & \\
\hline no $u /$ Swift $u$ & $0.87 \pm 0.84$ & $1.30 \pm 1.23$ & 0.14 & -0.9 & $0.28 \pm 0.21$ & $2.28 \pm 1.07$ & 1.13 & -1.0 & & \\
\hline
\end{tabular}

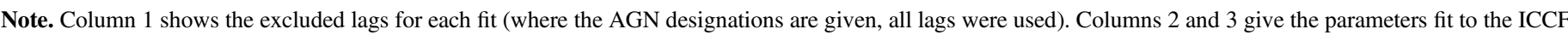

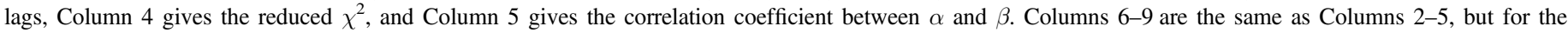

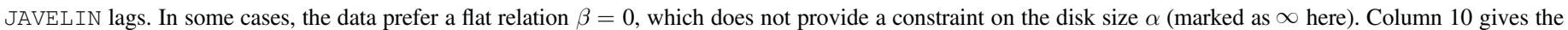

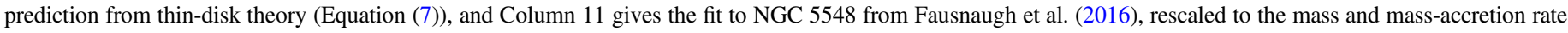
of these objects (see Section 5.3).

would also have to underestimated $\dot{m}_{\text {Edd }}$ by a factor of 1.4-4.0. As discussed in Section 4, the choice of bolometric correction may be responsible for part of this difference. Internal extinction, kinematic luminosity (i.e., energy loss in outflows), and advection may also be important, because these effects will cause the observed luminosity to underestimate the true energy generation rate and the inferred value of $\dot{m}_{\text {Edd }}$. Finally, the normalization of Equation (7) depends on the radiative efficiency $\eta$ and relative heating by irradiation from X-rays/ far-UV emission. The radiative efficiency must be less than 0.1 to increase the predicted size of $\tau_{0}$, which implies a counterrotating black hole relative to the disk and is a priori unlikely, while setting the heating term from irradiation to match that of viscous dissipation only increases $\tau_{0}$ by $\sim 10 \%$ (Fausnaugh et al. 2016). Given these uncertainties, there seems to be no problem accounting for the discrepancy in NGC $2617(\sim 40 \%)$. The factor of 4 discrepancy in MCG+08-11-011 is more difficult to account for, although a combination of effects may be able to explain the difference.

An even larger fluctuation (greater than a factor of 3) of $M_{\mathrm{BH}}$ beyond the estimate from Fausnaugh et al. (2017) might cause the discrepancies in disk size and $\dot{m}_{\text {Edd }}$ to vanish. Conversely, fluctuations of $M_{\mathrm{BH}}$ below these estimates would cause severe energy budget problems, by about an order of magnitude, compared to the optical luminosity. Uncertainty in the individual estimates of $M_{\mathrm{BH}}$ therefore limits our ability to assess the significance of any discrepancies with the thin disk model, and improving these uncertainties is a critical path forward.

These results are consistent with our findings in Section 4, where we showed that the accretion rates inferred from CREAM are formally larger than those estimated from the optical luminosity. As a reminder, CREAM finds discrepancies in $\dot{m}_{\text {Edd }}$ of a factor of 4.3 and 1.3 in MCG+08-11-011 and NGC 2617, respectively, although these estimates are statistically consistent with 1, given the large uncertainties. CREAM employs a physical model of the disk, which probably results in more accurate estimates of the accretion rate than using the fluxweighted mean radius in Equation (7). This seems to suggest that any discrepancy in disk size or $\dot{m}_{\text {Edd }}$ is less severe than indicated above. However, the values of $\chi^{2} /$ dof for the CREAM fits were somewhat larger than unity, which may indicate that the CREAM model does not adequately describe the data. Furthermore, the CREAM uncertainties are large because they again depend on the black hole mass.

If the disks are larger than expected from thin-disk theory, these results are similar to those from RM for the accretion disk in NGC 5548. Fausnaugh et al. (2016) find a disk in this object three times larger than the prediction of standard thin-disk theory. However, they assumed that the accretion rate was $10 \%$ 
of Eddington and that irradiation by high-energy emission contributed significant heating to the disk. Based on optical spectroscopy taken during the AGN STORM campaign (Pei et al. 2017), we measure the Eddington ratio of NGC 5548 in 2014 to be $5 \%$ (again taking $\dot{m}_{\text {Edd }}=10 \lambda L_{5100 \AA} / L_{\text {Edd }}$ ). Assuming that the X-rays/far-UV contribute negligible heating (as we did in Equation (7)), the disk in this object is a factor of 4.4 larger than thin-disk theory. As a comparison, we rescaled the large disk from NGC 5548 to the mass and mass-accretion rate of our targets using the same dependencies as in Equation (7). These comparisons are given in Table 8 and shown in Figure 5 by the dashed red lines. Our fits lie between the NGC 5548 result and the prediction from thin-disk theory. Unlike the case of NGC 5548, we are forced to extrapolate the lag-wavelength relation to far-UV wavelengths. Therefore, there are considerably larger uncertainties associated with our estimate of the disk's absolute size. However, the qualitative agreement (an accretion disk larger than predictions, by a factor of a few) is striking.

\section{Summary}

We have detected interband continuum lags in two Seyfert 1 galaxies, MCG+08-11-011 and NCG 2617. This adds one new object to the previous sample of two AGNs with secure measurements of accretion disk reverberation signals. We also compared the lags for NGC 2617 in 2014 to lags measured one year prior in 2013 by Shappee et al. (2014).

(i) We generally find longer lags at longer wavelengths, consistent with disk reprocessing models. The exceptions are the $u$-band data for in NGC 2617 and the $g$-band for $\mathrm{MCG}+08-11-011$. For NGC 2617, these longer lags are probably due to contamination by the Balmer continuum. The origin of the anomalous lag in MCG+08-11-011 is less clear, but it may be caused by a similar bias in the broadband filters that is not present in the spectroscopic continuum light curve.

(ii) The X-ray to UV/optical lag in NGC 2617 is $~ 2.6$ days, and there is substantially more structure in the X-ray light curve than the UV/optical light curves. This is inconsistent with standard reprocessing models where the $\mathrm{X}$-ray emitting corona directly irradiates the surrounding accretion disk. However, there is still some correlation between these light curves, suggesting a complicated relationship between the X-ray and UV/optical emission.

(iii) The lag-wavelength relations (for the UV/optical light curves) are consistent with the predictions for reprocessing in a standard geometrically thin disk. However, the inferred disk sizes are larger than these predictions by a factor of 3.3 in MCG+08-11-011 and 2.3 in NGC 2617.

(iv) These results may indicate that the observed optical luminosities underestimate the total energy generation (mass accretion) rates. Using the CREAM physical reprocessing model to fit the light curves, we find Eddington ratios larger than would be estimated from the optical luminosity, by a factor of 4.3 in $\mathrm{MCG}+08-11-011$ and a factor of 1.6 in NGC 2617. However, these differences are not statistically significant, considering uncertainty in the SMBH masses.

These results add to the growing body of evidence for additional structure and possibly physical processes in AGN accretion disks: the X-ray phenomenology indicates a more complicated situation than simple disk reprocessing, and there is tension between the sizes of the disks and standard models. However, the significance of this statement is limited by uncertainties in the SMBH masses. A direct means of expanding our work would be to improve the SMBH mass uncertainties, for example, by using the dynamical models of Pancoast et al. (2014) to estimate the individual virial factor $f$ of each object. We will pursue such an analysis for MCG+08-11011 and NGC 2617 in future work using our spectroscopic RM data (see Fausnaugh et al. 2017 for details). An alternative tactic, which has been adopted by the LCO AGN Key project, is to expand the sample size of RM-measured AGN disk sizes. This will improve the uncertainty on the mean AGN disk size, and potentially pin down departures from standard models. Finally, microlensing of strongly lensed quasars is the only other practical means of probing the accretion disks around SMBHs. Both microlensing and RM find similar disk sizes (e.g., Mosquera et al. 2013), and a thorough and systematic comparison of the microlensing and RM results is therefore warranted.

M.M.F. acknowledges financial support from a Presidential Fellowship awarded by The Ohio State University Graduate School. NSF grant AST-1008882 supported M.M.F., G.D.R., B.M.P., and R.W.P. M.C.B. gratefully acknowledges support through NSF CAREER grant AST-1253702 to Georgia State University. K.D.D. is supported by an NSF AAPF fellowship awarded under NSF grant AST-1302093. C.S.K. is supported by NSF grant AST-1515876. K.H. acknowledges support from STFC grant ST/M001296/1. This material is based in part upon work supported by the National Science Foundation (NSF) Graduate Research Fellowship Program under Grant No. DGE-0822215, awarded to C.B.H., and A.M.M. acknowledges the support of NSF grant AST-1211146. M.E. thanks the members of the Center for Relativistic Astrophysics at Georgia Tech, where he was based during the observing campaign, for their warm hospitality. J.S.S. acknowledges CNPq, National Council for Scientific and Technological Development, Brazil. J.T. acknowledges support from NSF grant AST-1411685. Work by S.V. Jr. is supported by the National Science Foundation Graduate Research Fellowship under Grant No. DGE-1343012. Work by W.Z. was supported by NSF grant AST-1516842. T.W.-S.H. is supported by the DOE Computational Science Graduate Fellowship, grant number DE-FG0297ER25308. E.R.C. and S.M.C. gratefully acknowledge the receipt of research grants from the National Research Foundation (NRF) of South Africa. T.T. acknowledges support by the National Science Foundation through grant AST1412315 "Collaborative Research: New Frontiers in Reverberation Mapping," and by the Packard Foundation through a Packard Research Fellowship. D.J.S. acknowledges support from NSF grants AST-1412504 and AST-1517649. A.J.B. and L.P. have been supported by NSF grant AST-1412693. B.J.S. is supported by NASA through Hubble Fellowship grant HF51348.001 awarded by the Space Telescope Science Institute, which is operated by the Association of Universities for Research in Astronomy, Inc., for NASA, under contract NAS 5-26555.

M.C.B. acknowledges support through grant HST GO-13816 from the Space Telescope Science Institute, which is operated 
by the Association of Universities for Research in Astronomy, Inc., under NASA contract NAS5-26555. This research has made use of the XRT Data Analysis Software (XRTDAS), developed under the responsibility of the ASI Science Data Center (ASDC), Italy. This work is based on observations obtained at the MDM Observatory, operated by Dartmouth College, Columbia University, Ohio State University, Ohio University, and the University of Michigan. This paper is partly based on observations collected at the Wise Observatory with the $\mathrm{C} 18$ telescope. The $\mathrm{C} 18$ telescope and most of its equipment were acquired with a grant from the Israel Space Agency (ISA) to operate a Near-Earth Asteroid Knowledge Center at Tel Aviv University. The Fountainwood Observatory would like to thank the HHMI for its support of science research for undergraduate students at Southwestern University. This research has made use of NASA's Astrophysics Data System, as well as the NASA/IPAC Extragalactic Database (NED) which is operated by the Jet Propulsion Laboratory,
California Institute of Technology, under contract with the National Aeronautics and Space Administration.

Facilities: McGraw-Hill, HST, Wise Observatory, Fountainwood Observatory BYU:0.9m, CrAO:0.7 m, WIRO, LCO, LCOGT, SSO:1m, Swift.

Software: Astropy (Astropy Collaboration et al. 2013), IRAF (Tody 1986), Matplotlib (Hunter 2007), Numpy (van der Walt et al. 2011), Scipy (Oliphant 2007).

\section{Appendix}

Here we show the detailed results of the CREAM fits, including interpolated light curves, transfer functions, and the inferred driving light curves. Figure 6 shows the results for MCG+08-11-011. Figure 7 shows the fits to the Swift data (X-ray, near-UV, and optical light curves) for NGC 2617, and Figure 8 shows the fits to the ground-based data for NGC 2617. Note that all data for NGC 2617 were fit simultaneously.
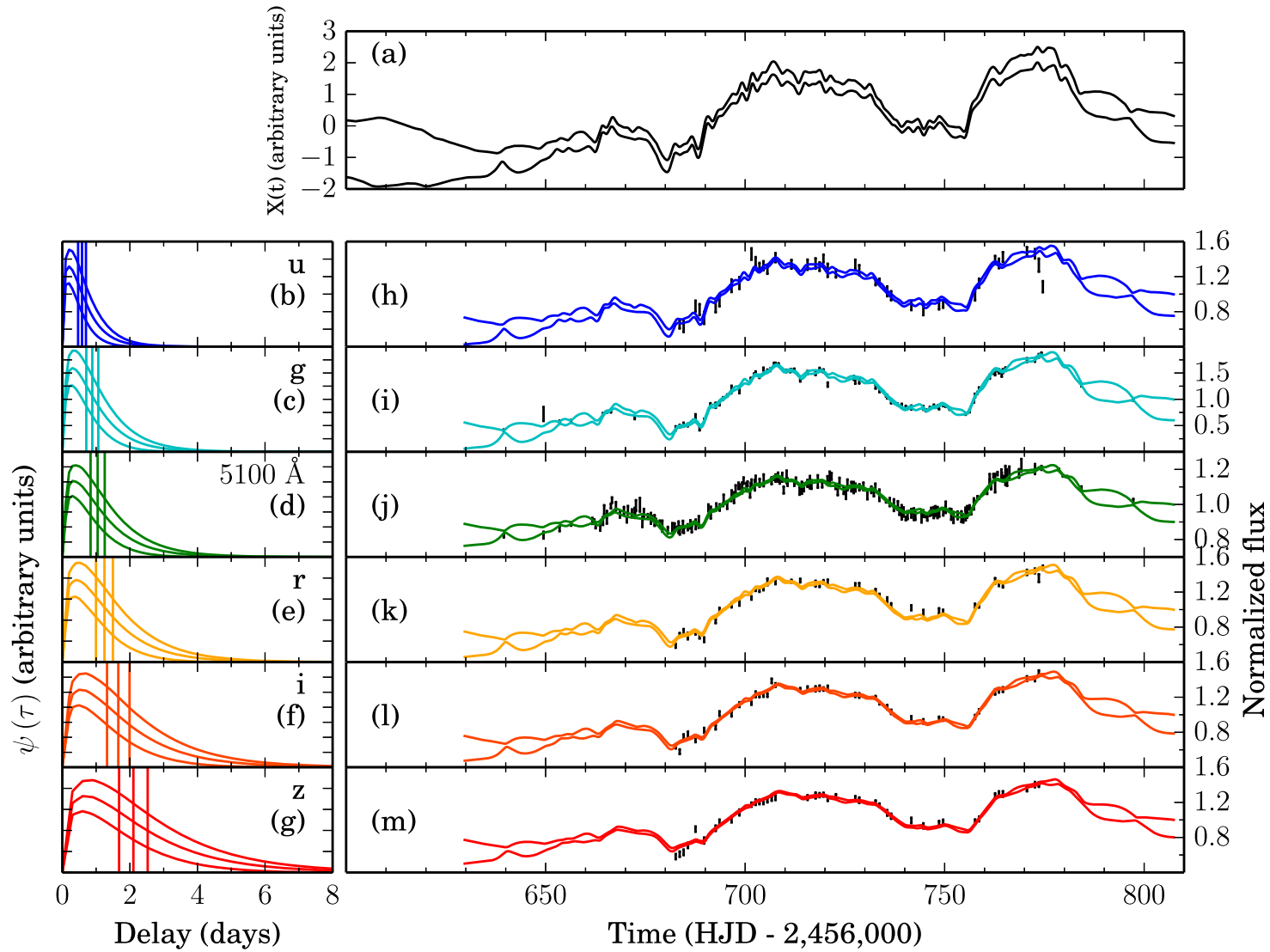

Figure 6. CREAM fits to the $u$ - through $z$-band light curves for MCG+08-11-011. Panel (a) shows the inferred driving light curve for the disk continuum variations. Panels $(b-g)$ show the inferred transfer functions where the middle, lower, and upper curves correspond to the mean and $1 \sigma$ uncertainties. The vertical lines denote the mean lags. Panels $(\mathrm{h}-\mathrm{m})$ show the light curves and uncertainty envelopes. 

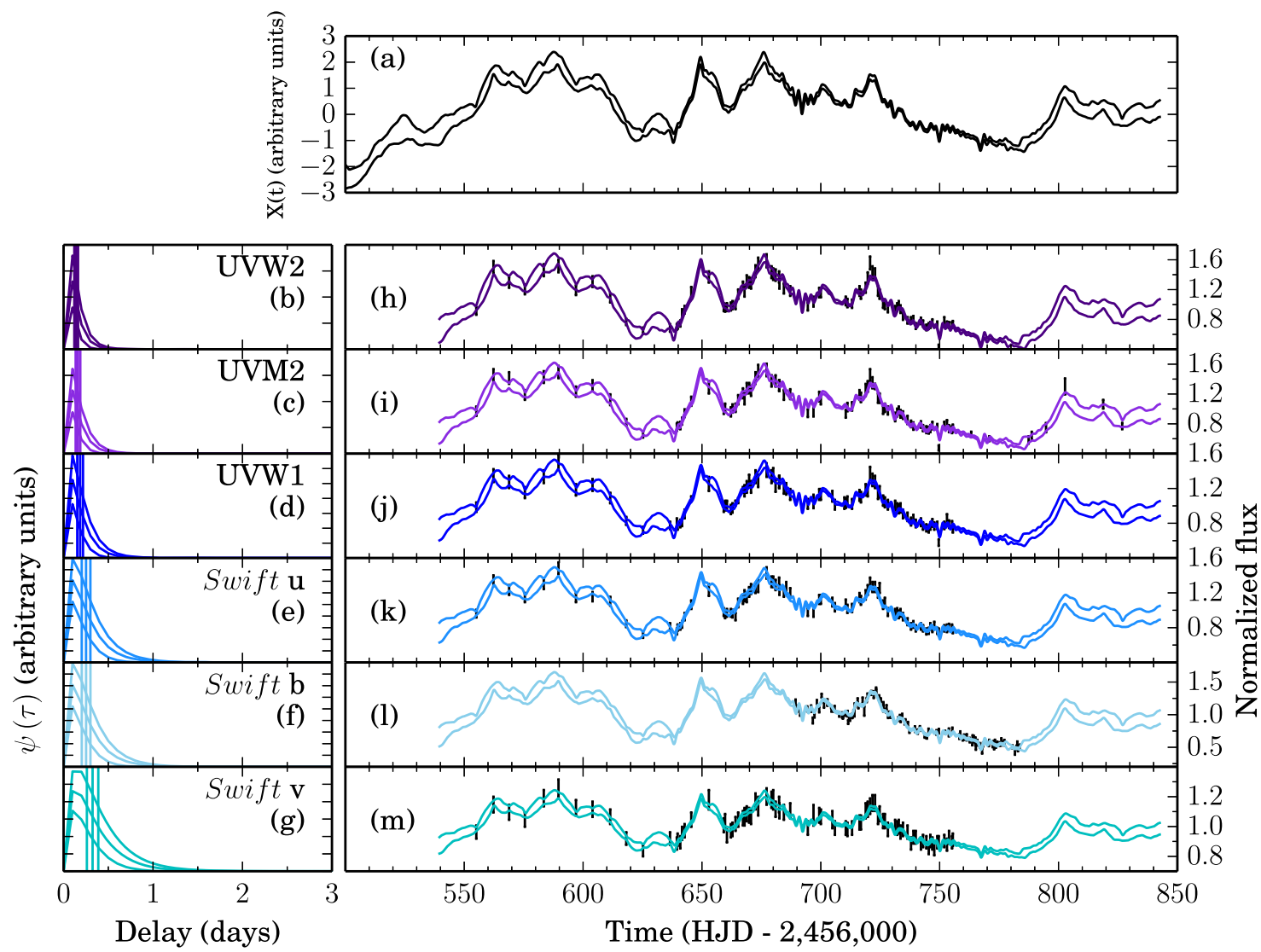

Figure 7. Same as Figure 6, but for Swift data of NGC 2617.
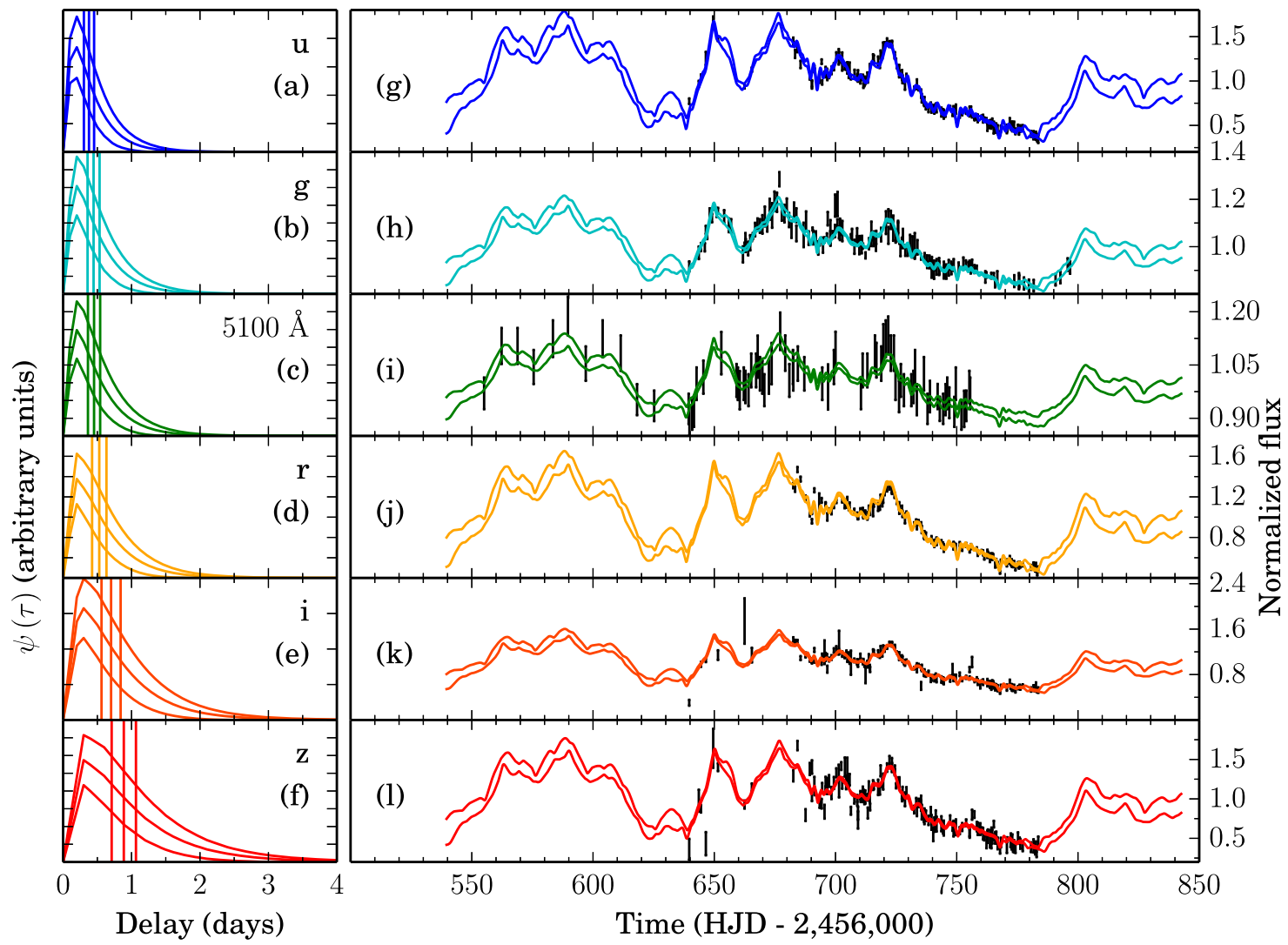

Figure 8. Same as Figure 6, but for ground-based data of NGC 2617. 


\section{ORCID iDs}

Keith Horne (1) https://orcid.org/0000-0003-1728-0304 C. S. Kochanek ㄴ) https://orcid.org/0000-0001-6017-2961

M. C. Bentz (1) https://orcid.org/0000-0002-2816-5398

C. J. Grier (i) https://orcid.org/0000-0001-9920-6057

D. Grupe iㅏ https://orcid.org/0000-0002-9961-3661

R. W. Pogge (1) https://orcid.org/0000-0003-1435-3053

A. J. Barth (ib https://orcid.org/0000-0002-3026-0562

Thomas G. Beatty (1) https://orcid.org/0000-0002-9539-4203

T. A. Boroson (i) https://orcid.org/0000-0001-9481-1805

Jonathan S. Brown (1) https://orcid.org/0000-0002-1885-6419

M. S. Brotherton (1) https://orcid.org/0000-0002-1207-0909

C. T. Coker (1) https://orcid.org/0000-0002-9954-7887

S. M. Crawford (1) https://orcid.org/0000-0002-8969-5229

K. V. Croxall (i) https://orcid.org/0000-0002-5258-7224

Michael Eracleous (ㅈ) https://orcid.org/0000-0002-3719-940X

C. B. Henderson (1) https://orcid.org/0000-0001-8877-9060

T. W.-S. Holoien (1) https://orcid.org/0000-0001-9206-3460

Miao Li (1) https://orcid.org/0000-0003-0773-582X

Zhiyuan Ma (1) https://orcid.org/0000-0003-3270-6844

Dale Mudd (1) https://orcid.org/0000-0003-2371-4121

Christopher A. Onken (i) https://orcid.org/0000-0003-

0017-349X

A. Pancoast (ㄱ) https://orcid.org/0000-0003-1065-5046

Matthew T. Penny (1) https://orcid.org/0000-0001-7506-5640

David J. Sand (i) https://orcid.org/0000-0003-4102-380X

Jaderson S. Schimoia (1) https://orcid.org/0000-0002-

5640-6697

B. J. Shappee (1) https://orcid.org/0000-0003-4631-1149

Garrett Somers (i) https://orcid.org/0000-0002-9322-0314

M. Spencer (1) https://orcid.org/0000-0003-1240-1939

Jamie Tayar (1) https://orcid.org/0000-0002-4818-7885

T. Treu (1) https://orcid.org/0000-0002-8460-0390

Stefano Valenti $\odot$ https://orcid.org/0000-0001-8818-0795

S. Villanueva, Jr. (1) https://orcid.org/0000-0001-6213-8804

C. Villforth (10) https://orcid.org/0000-0002-8956-6654

H. Winkler (1) https://orcid.org/0000-0003-2662-0526

\section{References}

Abazajian, K. N., Adelman-McCarthy, J. K., Agüeros, M. A., et al. 2009, ApJS, 182, 543

Abramowicz, M. A., Czerny, B., Lasota, J. P., \& Szuszkiewicz, E. 1988, ApJ, 332,646

Alard, C., \& Lupton, R. H. 1998, ApJ, 503, 325

Arévalo, P., Uttley, P., Kaspi, S., et al. 2008, MNRAS, 389, 1479

Arévalo, P., Uttley, P., Lira, P., et al. 2009, MNRAS, 397, 2004

Astropy Collaboration, Robitaille, T. P., Tollerud, E. J., et al. 2013, A\&A, 558, A33

Balbus, S. A., \& Hawley, J. F. 1998, RvMP, 70, 1

Begelman, M. C., Armitage, P. J., \& Reynolds, C. S. 2015, ApJ, 809, 118

Begelman, M. C., \& Silk, J. 2017, MNRAS, 464, 2311

Blackburne, J. A., Pooley, D., Rappaport, S., \& Schechter, P. L. 2011, ApJ, 729,34

Blaes, O. 2014, SSRv, 183, 21

Blandford, R. D., \& McKee, C. F. 1982, ApJ, 255, 419

Breedt, E., Arévalo, P., McHardy, I. M., et al. 2009, MNRAS, 394, 427

Breedt, E., McHardy, I. M., Arévalo, P., et al. 2010, MNRAS, 403, 605

Brosch, N., Polishook, D., Shporer, A., et al. 2008, Ap\&SS, 314, 163

Brown, T. M., Baliber, N., Bianco, F. B., et al. 2013, PASP, 125, 1031

Buisson, D. J. K., Lohfink, A. M., Alston, W. N., \& Fabian, A. C. 2017, MNRAS, 464, 3194

Burbidge, E. M. 1967, ARA\&A, 5, 399

Burrows, D. N., Hill, J. E., Nousek, J. A., et al. 2005, SSRv, 120, 165

Cackett, E. M., Horne, K., \& Winkler, H. 2007, MNRAS, 380, 669
Capellupo, D. M., Netzer, H., Lira, P., Trakhtenbrot, B., \& Mejía-Restrepo, J. 2015, MNRAS, 446, 3427

Collier, S. J., Horne, K., Kaspi, S., et al. 1998, ApJ, 500, 162

Davidson, K., \& Netzer, H. 1979, RvMP, 51, 715

De Rosa, G., Peterson, B. M., Ely, J., et al. 2015, ApJ, 806, 128

Edelson, R., Gelbord, J., Cackett, E., et al. 2017, ApJ, 840, 41

Edelson, R., Gelbord, J. M., Horne, K., et al. 2015, ApJ, 806, 129

Elvis, M., Wilkes, B. J., McDowell, J. C., et al. 1994, ApJS, 95, 1

Fausnaugh, M. M. 2017, PASP, 129, 024007

Fausnaugh, M. M., Denney, K. D., Barth, A. J., et al. 2016, ApJ, 821, 56

Fausnaugh, M. M., Grier, C. J., Bentz, M. C., et al. 2017, ApJ, 840, 97

Gardner, E., \& Done, C. 2017, MNRAS, 470, 3591

Gaskell, C. M., \& Peterson, B. M. 1987, ApJS, 65, 1

Giustini, M., Costantini, E., De Marco, B., et al. 2017, A\&A, 597, A66

Gliozzi, M., Papadakis, I. E., Grupe, D., Brinkmann, W. P., \& Räth, C. 2017, MNRAS, 464, 3955

Goad, M. R., \& Korista, K. T. 2014, MNRAS, 444, 43

Haardt, F., \& Maraschi, L. 1991, ApJL, 380, L51

Horne, K. 1994, in ASP Conf. Ser. 69, Reverberation Mapping of the BroadLine Region in Active Galactic Nuclei, ed. P. M. Gondhalekar, K. Horne, \& B. M. Peterson (San Francisco, CA: ASP), 23

Hunter, J. D. 2007, CSE, 9, 90

Jiang, Y.-F., Green, P. J., Greene, J. E., et al. 2017, ApJ, 836, 186

Jiang, Y.-F., Stone, J. M., \& Davis, S. W. 2014, ApJ, 784, 169

Jiménez-Vicente, J., Mediavilla, E., Kochanek, C. S., et al. 2014, ApJ, 783, 47

Kishimoto, M., Antonucci, R., Blaes, O., et al. 2008, Natur, 454, 492

Kishimoto, M., Antonucci, R., Boisson, C., \& Blaes, O. 2004, MNRAS, 354,1065

Kokubo, M. 2015, MNRAS, 449, 94

Kokubo, M. 2016, PASJ, 68, 52

Korista, K. T., \& Goad, M. R. 2001, ApJ, 553, 695

Kozłowski, S. 2017, A\&A, 597, A128

Krolik, J. H., Horne, K., Kallman, T. R., et al. 1991, ApJ, 371, 541

LaMassa, S. M., Cales, S., Moran, E. C., et al. 2015, ApJ, 800, 144

Lira, P., Arévalo, P., Uttley, P., McHardy, I. M. M., \& Videla, L. 2015, MNRAS, 454, 368

Luo, C., \& Liang, E. P. 1998, ApJ, 498, 307

MacLeod, C. L., Ross, N. P., Lawrence, A., et al. 2016, MNRAS, 457, 389

Maoz, D., Markowitz, A., Edelson, R., \& Nandra, K. 2002, AJ, 124, 1988

Marshall, K., Ryle, W. T., \& Miller, H. R. 2008, ApJ, 677, 880

McHardy, I. M., Cameron, D. T., Dwelly, T., et al. 2014, MNRAS, 444, 1469

McHardy, I. M., Connolly, S. D., Peterson, B. M., et al. 2016, AN, 337, 500

Morgan, C. W., Kochanek, C. S., Morgan, N. D., \& Falco, E. E. 2010, ApJ, 712,1129

Mosquera, A. M., Kochanek, C. S., Chen, B., et al. 2013, ApJ, 769, 53

Narayan, R., \& Yi, I. 1995, ApJ, 452, 710

Nealon, R., Price, D. J., \& Nixon, C. J. 2015, MNRAS, 448, 1526

Nenkova, M., Sirocky, M. M., Nikutta, R., Ivezić, Ž., \& Elitzur, M. 2008, ApJ, 685,160

Noble, S. C., Krolik, J. H., Schnittman, J. D., \& Hawley, J. F. 2011, ApJ, 743,115

Oknyansky, V. L., Gaskell, C. M., Huseynov, N. A., et al. 2017, MNRAS, 467, 1496

Oliphant, T. E. 2007, CSE, 9, 10

Onken, C. A., Ferrarese, L., Merritt, D., et al. 2004, ApJ, 615, 645

Page, D. N., \& Thorne, K. S. 1974, ApJ, 191, 499

Pancoast, A., Brewer, B. J., Treu, T., et al. 2014, MNRAS, 445, 3073

Pei, L., Fausnaugh, M. M., Barth, A. J., et al. 2017, ApJ, 837, 131

Pereyra, N. A., Vanden Berk, D. E., Turnshek, D. A., et al. 2006, ApJ, 642, 87

Peterson, B. M. 1993, PASP, 105, 247

Peterson, B. M. 2014, SSRv, 183, 253

Peterson, B. M., Ferrarese, L., Gilbert, K. M., et al. 2004, ApJ, 613, 682

Rees, M. J. 1984, ARA\&A, 22, 471

Reis, R. C., \& Miller, J. M. 2013, ApJL, 769, L7

Reynolds, C. S., \& Nowak, M. A. 2003, PhR, 377, 389

Roming, P. W. A., Kennedy, T. E., Mason, K. O., et al. 2005, SSRv, 120, 95

Runnoe, J. C., Brotherton, M. S., \& Shang, Z. 2012, MNRAS, 422, 478

Runnoe, J. C., Cales, S., Ruan, J. J., et al. 2016, MNRAS, 455, 1691

Sądowski, A. 2016, MNRAS, 459, 4397

Sądowski, A., \& Narayan, R. 2015, MNRAS, 454, 2372

Sądowski, A., Narayan, R., McKinney, J. C., \& Tchekhovskoy, A. 2014, MNRAS, 439, 503

Schmidt, K. B., Rix, H.-W., Shields, J. C., et al. 2012, ApJ, 744, 147

Schnittman, J. D., \& Krolik, J. H. 2013, ApJ, 777, 11

Schnittman, J. D., Krolik, J. H., \& Noble, S. C. 2013, ApJ, 769, 156 
Schnittman, J. D., Krolik, J. H., \& Noble, S. C. 2016, ApJ, 819, 48 Sergeev, S. G., Doroshenko, V. T., Golubinskiy, Y. V., Merkulova, N. I., \& Sergeeva, E. A. 2005, ApJ, 622, 129

Shakura, N. I., \& Sunyaev, R. A. 1973, A\&A, 24, 337

Shankar, F., Calderone, G., Knigge, C., et al. 2016, ApJL, 818, L1

Shappee, B. J., Prieto, J. L., Grupe, D., et al. 2014, ApJ, 788, 48

Shields, G. A. 1978, Natur, 272, 706

Skielboe, A., Pancoast, A., Treu, T., et al. 2015, MNRAS, 454, 144

Starkey, D., Horne, K., Fausnaugh, M. M., et al. 2017, ApJ, 835, 65

Starkey, D. A., Horne, K., \& Villforth, C. 2016, MNRAS, 456, 1960

Suganuma, M., Yoshii, Y., Kobayashi, Y., et al. 2006, ApJ, 639, 46

Telfer, R. C., Zheng, W., Kriss, G. A., \& Davidsen, A. F. 2002, ApJ, 565, 773

Tody, D. 1986, Proc. SPIE, 627, 733

Troyer, J., Starkey, D., Cackett, E. M., et al. 2016, MNRAS, 456, 4040
Turner, T. J., Miller, L., George, I. M., \& Reeves, J. N. 2006, A\&A, 445, 59 Uttley, P., Edelson, R., McHardy, I. M., Peterson, B. M., \& Markowitz, A. 2003, ApJL, 584, L53

Valenti, S., Sand, D. J., Barth, A. J., et al. 2015, ApJL, 813, L36

van der Walt, S., Colbert, S. C., \& Varoquaux, G. 2011, CSE, 13, 22

Vanden Berk, D. E., Richards, G. T., Bauer, A., et al. 2001, AJ, 122, 549

Vazquez, B., Galianni, P., Richmond, M., et al. 2015, ApJ, 801, 127

Veilleux, S., \& Osterbrock, D. E. 1987, ApJS, 63, 295

Weedman, D. W. 1977, ARA\&A, 15, 69

Welsh, W. F. 1999, PASP, 111, 1347

White, R. J., \& Peterson, B. M. 1994, PASP, 106, 879

Wilhite, B. C., Vanden Berk, D. E., Kron, R. G., et al. 2005, ApJ, 633, 638 Zu, Y., Kochanek, C. S., Kozłowski, S., \& Udalski, A. 2013, ApJ, 765, 106

Zu, Y., Kochanek, C. S., \& Peterson, B. M. 2011, ApJ, 735, 80 